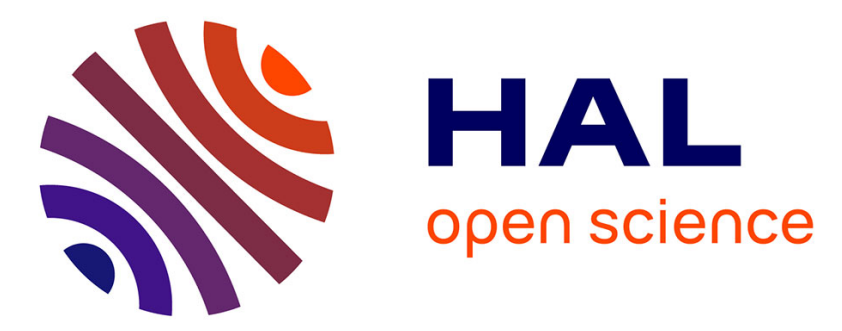

\title{
Toujours plus, toujours mieux? Effet contre-intuitif de l'évaluation des attributs environnementaux du produit par le consommateur
}

\author{
Eline Jongmans, Alain Jolibert, Julie Irwin
}

\section{> To cite this version:}

Eline Jongmans, Alain Jolibert, Julie Irwin. Toujours plus, toujours mieux? Effet contre-intuitif de l'évaluation des attributs environnementaux du produit par le consommateur. 2014, 48 p. halshs01185784

\section{HAL Id: halshs-01185784 \\ https://shs.hal.science/halshs-01185784}

Submitted on 26 Jan 2016

HAL is a multi-disciplinary open access archive for the deposit and dissemination of scientific research documents, whether they are published or not. The documents may come from teaching and research institutions in France or abroad, or from public or private research centers.
L'archive ouverte pluridisciplinaire HAL, est destinée au dépôt et à la diffusion de documents scientifiques de niveau recherche, publiés ou non, émanant des établissements d'enseignement et de recherche français ou étrangers, des laboratoires publics ou privés. 


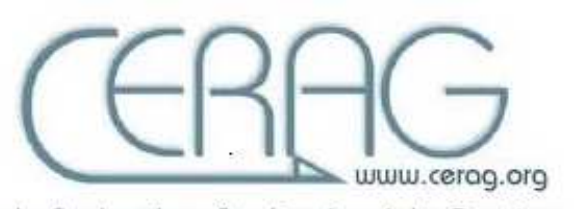

Centre d'Etudes et de Recherches Appliquées d̀ la Gestion_U.M.R. C.N.R.S. 5820

\section{CAHIER DE RECHERCHE $n^{\circ} 2014-02$ E3}

Toujours plus, toujours mieux? Effet contre-intuitif de l'évaluation des attributs environnementaux du produit par le consommateur

JONGMANS Eline

JOLIBERT Alain

IRWIN Julie 
Toujours plus, toujours mieux ? Effet contre-intuitif de l'évaluation des attributs environnementaux du produit par le consommateur

\author{
Éline Jongmans \\ Doctorante en Marketing \\ Laboratoire CERAG - UMR 5820 du CNRS \\ Université de Grenoble-Alpes \\ eline.jongmans@upmf-grenoble.fr
}

\author{
Alain Jolibert \\ Professeur des Universités \\ Laboratoire CERAG - UMR 5820 du CNRS \\ INSEEC Business School \\ alain.jolibert@upmf-grenoble.fr
}

Julie Irwin

Professeur de Marketing

McCombs School of Business

Université du Texas à Austin

julie.irwin@mccombs.utexas.edu 


\section{Toujours plus, toujours mieux? Effet contre-intuitif de l'évaluation des attributs environnementaux du produit par le consommateur}

\section{RÉSUMÉ}

L'objectif de cet article est d'étudier l'influence du nombre d'attributs environnementaux d'un produit de consommation sur le poids associé à ces attributs. Les résultats de deux expérimentations montrent que les consommateurs tendent à systématiquement sous-évaluer les attributs environnementaux (certifiés ou non) lorsqu'ils sont présentés ensemble, par rapport à une évaluation des mêmes attributs présentés séparément. Cet effet non rationnel, appelé effet d'inclusion, est plus fort pour les individus davantage préoccupés par l'environnement et impliqués envers les écolabels. Les conclusions de cette étude remettent en question l'intérêt de cumuler de tels attributs pour les consommateurs de produits pro-environnementaux.

Mots-clés : consommation verte, marketing et environnement, évaluation de produit, attribut environnemental, effet d'inclusion, préoccupation pour l'environnement, implication envers les écolabels

\section{ABSTRACT}

The objective of this paper is to study the influence of the number of environmental attributes on the weight given to these attributes for a consumption product. The results of two experiments show that certified or non-certified environmental attributes on the same consumption product are weighted less (more) by consumers when they are presented jointly (separately). This non-rational effect, called the embedding effect, is stronger for individuals deeply concerned about the environment and involved with ecolabels. The outcome of this study challenges the interest of accumulating attributes when dealing with consumers of environmentally friendly products.

Keywords: green consumption, marketing and environment, product valuation, environmental attribute, embedding effect, environmental concern, involvement with ecolabels 


\section{INTRODUCTION}

Ces vingt dernières années, la nécessité de protéger l'environnement pour assurer un développement durable aux générations futures est apparue, impliquant la responsabilité des acteurs du marché (i.e. consommateurs, managers, État). Cette prise de conscience a notamment conduit à la «consommation verte », nuisant le moins possible à l'environnement ou lui étant bénéfique (Steg et Vlek, 2009), et à la consommation de produits pro-environnementaux (Mazar et Zhong, 2010). Ces derniers possèdent des attributs renvoyant à des valeurs sociétales ou environnementales non marchandes comme la protection de l'environnement. En France, la diffusion d'écolabels certifiés a doublé entre 2008 et 2011 sur les produits de consommation (SNDD, 2012). Une étude réalisée en Amérique du Nord montre que l'offre de produits « verts » (certifiés ou non-certifiés) a augmenté de $73 \%$ entre 2009 et 2010 (TerraChoice, 2010). Ces évolutions sont cependant à double tranchant. D'une part, elles répondent à une demande accrue des consommateurs. D'autre part, ceux-ci peuvent être perdus dans la pléthore des labels et mentions auto-déclarées (e.g. nom de marque pro-environnementale ; Comas et Seifert, 2012). Les directeurs marketing et chefs de produit ont donc besoin d'évaluer l'intérêt d'inclure ces attributs, souvent contraignants en temps et en investissement.

Dès les années 1970 (Henion, 1972), des travaux ont été consacrés aux profils des consommateurs sensibles aux attributs environnementaux. Ils ont notamment étudié le lien entre leurs valeurs individuelles et leur sensibilité plus importante à ces attributs (Brécard et alii, 2009; Grunert et Juhl, 1995), ou se sont intéressés à la capacité à réaliser un surprix (premium price) en utilisant un attribut environnemental (Loureiro, McCluskey et Mittelhammer, 2002; Sedjo et Swallow, 2002). Par contre, peu d'études ont porté sur les effets contre-intuitifs (i.e. biais systématique remettant en cause les modèles normatifs) des attributs environnementaux pour des produits de consommation (Irwin et Spira, 1997 ; Luchs et alii, 2010). Pour Darby et Karni (1973), les attributs liés à la protection de l'environnement (i.e. attributs environnementaux) sont considérés comme des attributs de croyance car en présence d'une mention auto-déclarée (i.e. non-certifiée), les acheteurs ne disposent pas des connaissances nécessaires pour vérifier l'information. Seul le producteur connaît la vraie qualité environnementale d'un produit (Bougherara et Piguet, 2008; Loureiro, McCluskey et Mittelhammer, 2002). Pour réduire cette asymétrie d'information, des écolabels officiels (i.e. certifiés par un organisme tiers et indépendant), ont été développés. Ceux-ci restent des attributs de croyance mais ils permettent aux consommateurs de mieux appréhender la qualité environnementale du produit avant l'achat. Cette double caractéristique (attribut de croyance certifié vs. non-certifié) complique l'évaluation des 
attributs environnementaux par les consommateurs. Elle conduit à s'interroger sur la manière dont ces derniers intègrent la présence d'un seul ou de plusieurs de ces attributs. Dans le cas d'attributs environnementaux non certifiés, Irwin et Spira (1997) mettent en évidence un effet contre-intuitif, qualifié d'effet d'inclusion. Il se caractérise par une évaluation systématiquement plus faible lorsque plusieurs attributs environnementaux sont présentés ensemble plutôt que présentés séparément. Dans la lignée d'Irwin et Spira (1997), nous étudions l'impact d'attributs environnementaux sur l'évaluation d'un produit de consommation selon leur nombre (1 vs. 2), en tenant compte de leur nature (attribut certifié vs. non-certifié) et des croyances environnementales du répondant. Un attribut environnemental certifié correspond dans ce travail à un écolabel, défini comme « une étiquette de produit communiquant une caractéristique environnementale d'un bien ou d'un service utilisant une certification par un organisme tiers et indépendant » (adapté de Kitira, 2009).

Par rapport aux études précédentes, ce travail comporte trois originalités principales : (1) déterminer si l'utilisation de plusieurs attributs environnementaux (certifié vs. non-certifié) est utile ou bien superflue. Nous nous attachons à mieux cerner l'effet d'inclusion, qui contrecarre une démarche marketing consistant à choisir un positionnement pro-environnemental et à multiplier les attributs environnementaux sur un même produit ; (2) prendre en compte la présence (ou non) d'une certification des attributs environnementaux, actuellement en fort développement sur les produits. La mise en évidence de l'effet d'inclusion pour des attributs environnementaux certifiés, n'a jamais été réalisée jusqu'à présent ; (3) étudier le rôle modérateur de la sensibilité individuelle à l'égard de l'environnement (i.e. préoccupation pour l'environnement et implication envers les écolabels) pour améliorer la compréhension du phénomène. À ces fins, nous utilisons deux expérimentations. La première est destinée à mettre en évidence l'effet d'inclusion pour des attributs certifiés ou non-certifiés et le rôle modérateur de la préoccupation pour l'environnement sur cet effet d'inclusion, tandis que la seconde vise à mettre en évidence un effet d'inclusion pour des attributs certifiés et teste un autre modérateur, l'implication envers les écolabels, sur cet effet contre-intuitif.

\section{L'EFFET D'INCLUSION ET LES ATTRIBUTS ENVIRONNEMENTAUX}

\section{L'évaluation de produits avec attribut environnemental}

Trois principaux courants théoriques existent pour étudier les mécanismes d'évaluation d'un produit (Pham, 1996). Le premier est l'approche normative des comportements issue de la litté- 
rature économique, qui postule que, dès qu'un individu affecte une valeur non nulle à une quantité de biens, il devrait donner une valeur supérieure à une plus grande quantité de biens ; c'est le principe de variation de grandeur (Fischhoff et alii, 1993). Le deuxième courant est l'approche issue de la psychologie, qui cherche à établir des modèles représentant les processus de décision des individus en situation réelle. Elle comprend notamment le modèle multi-attribut de Fisbein et Ajzen (1975) mettant en évidence une relation entre croyances, attitude et intention de comportement. La troisième approche correspond à la théorie du comportement décisionnel, située à la jonction des deux premières. Elle s'intéresse aux biais systématiques ou effets contre-intuitifs qui apparaissent entre les prédictions des modèles normatifs et les comportements réels observés (Einhorn et Hogarth, 1981).

Un attribut environnemental est une caractéristique positive d'un produit relative à la protection de l'environnement. Nous distinguons deux types d'attributs : l'attribut certifié (i.e. attribut pour lequel l'information annoncée est vérifiée par un organisme tiers et indépendant) et l'attribut non-certifié (i.e. attribut autoproclamé par l'entreprise sans vérification externe). Lors de l'évaluation d'un produit comportant des attributs environnementaux, des compromis parfois délicats doivent être faits entre les attributs présents (e.g. prix $v s$. protection de l'environnement) (Ehrich et Irwin, 2005), produisant une source de biais systématiques. L'approche normative qui prédit que plus le poids d'un attribut est grand, meilleure est son évaluation est ainsi remise en question. La théorie du comportement décisionnel semble donc pertinente pour expliquer les mécanismes sous-jacents et les biais systématique liés à l'évaluation de produits avec attributs environnementaux.

\section{L'effet d'inclusion et ses mécanismes sous-jacents}

L'effet d'inclusion (embedding effect ou part-whole bias) est un effet de contexte mis en évidence lors de l'évaluation contingente des biens publics environnementaux (Kahneman et Knetsch, 1992a). L'évaluation contingente est une méthode de «valorisation par révélation directe des préférences individuelles fondées sur un marché hypothétique » (Le Gall-Ely et Robert-Demontrond, 2005, p. 13), sa spécificité est de recourir à un protocole explicatif de la tâche d'évaluation permettant l'évaluation de bien sans valeur marchande. Un exemple souvent repris pour illustrer l'effet d'inclusion est le travail de Desvousges et alii (1992) qui demandent le consentement à payer pour sauver 2000, 20000 ou 200000 oiseaux et qui obtiennent des réponses très proches et non significativement différentes ( $\$ 80, \$ 78$ et $\$ 88$, respectivement). La spécificité de renvoyer à des valeurs non marchandes, potentiellement importantes pour les in- 
dividus, rend l'attribut environnemental difficile à évaluer et peut conduire à l'apparition de l'effet d'inclusion (Kahneman et Knetsch, 1992a). Ce dernier survient lorsque le poids global donné à deux attributs environnementaux présents conjointement sur un produit est inférieur au poids global de ces deux attributs évalués seuls sur un produit (Irwin et Spira, 1997).

L'effet d'inclusion a suscité de nombreuses polémiques dans la littérature économique (Carson, 1997 ; Harrison, 1992 ; Smith, 1992), notamment en raison de la remise en cause de l'approche normative qu'il induit. A priori, l'évaluation des biens ou attributs devrait suivre le principe de variation de grandeur. Or, de nombreuses études contestent cette approche et montrent un effet d'inclusion pour des produits variés. Le Tableau 1 synthétise les principaux travaux consacrés à l'effet d'inclusion entre 1992 et 2011. Il montre la diversité des définitions de l'effet d'inclusion, principalement en économie, et le faible nombre d'études dont il fait l'objet en marketing. Un certain nombre de travaux en marketing étudient d'autres effets contre-intuitifs liés au contexte de prise de décision (e.g. Friedrich et alii, 1999; Chernev et Gal 2010 ; Urminsky et Kivetz, 2011).

\section{(Insérer Tableau 1)}

La première explication motivationnelle sous-jacente à l'effet d'inclusion, initialement proposée par Kahneman et Knetsch (1992a), est l'expression de valeurs idéologiques et non-économiques lors de l'évaluation. Ces valeurs, qui reflètent l'engagement moral des répondants, sont déconnectées de la valeur économique des produits et conduisent à l'insensibilité à la grandeur ou au nombre. Un seul attribut environnemental apposé sur un produit suffit à satisfaire l'engagement du consommateur envers la cause environnementale. Les individus « achètent » de la satisfaction morale. En économie, cet effet sous-jacent est appelé l'altruisme égocentré ou impur (warm glow) (Andreoni, 1989, 1990 ; Kahneman et Knetsch, 1992a). Il se produit si le répondant perçoit une forme d'utilité dans l'acte de donner ou dans la conscience qu'il joue son rôle en donnant pour un bien public, indépendamment de la grandeur du bien évalué (Shiell et Gold, 2002). La deuxième explication motivationnelle de l'effet d'inclusion, en accord avec la précédente, découle de la nature même de l'attribut environnemental. Souvent, ce dernier est lié à des valeurs dites «protégées » (Baron et Ritov, 2009 ; Baron et Spranca, 1997) pour lesquelles les individus n'arrivent pas facilement à faire de compromis (Irwin et Naylor, 2009), comme celles liées à la nature environnementale ou à la vie humaine (Ritov et Baron, 1999). Ces valeurs, insensibles à la grandeur de l'objet évalué, peuvent conduire les individus à donner une réponse biaisée en cas de compromis. Par exemple, lors d'une étude sur le montant que les consommateurs seraient prêts à payer pour une cause environnementale (Baron et Spranca, 1997), certains 
proposent $0 \$$ ou ne répondent pas, parce qu'ils n'arrivent pas à donner un prix à l'environnement.

Une explication cognitive de l'effet d'inclusion peut également compléter les explications motivationnelles conduisant à une insensibilité au nombre des attributs (Irwin et Spira, 1997). Un attribut environnemental conduit globalement au même degré de satisfaction morale qu'un autre. De ce point de vue deux attributs sont substituables. Un seul attribut environnemental pourrait donc suffire aux consommateurs à catégoriser le produit comme pro-environnemental, ce qui conduirait à une insensibilité au nombre lors de l'évaluation de plusieurs attributs.

Un engagement moral important envers la cause (ici environnementale) peut conduire à un effet d'inclusion (Irwin et Spira, 1997). Le poids donné à l'attribut environnemental, considéré comme une contribution à la cause environnementale, est en partie un signal de la force de sa croyance morale (Kahneman et Knetsch, 1992a). Nous postulons que l'évaluation des attributs environnementaux est sujette à l'effet d'inclusion, conduisant à une insensibilité au nombre d'attributs présents sur le produit et remettant en cause l'intérêt d'ajouter un attribut environnemental sur un produit. Dans cette étude, nous proposons donc l'hypothèse suivante :

$\mathrm{H} 1$ : Le poids global de deux attributs environnementaux présents conjointement sur un produit est moins important que le poids global des deux attributs environnementaux présentés seuls sur un produit.

\section{L'effet d'inclusion et ses modérateurs}

Plus l'engagement moral du consommateur envers la cause est fort, plus il l'exprimera lors de l'évaluation du produit, indépendamment du nombre d'attributs environnementaux. Irwin et Spira (1997) évoquent spécifiquement l'hypothèse d'une modération potentielle de l'effet d'inclusion par les croyances environnementales. Plus précisément, les individus pour lesquels la cause environnementale est importante devraient être plus fortement sujets à l'effet d'inclusion. Pour cela, nous proposons d'étudier le rôle modérateur de deux variables individuelles liées à la cause environnementale sur l'effet d'inclusion: la préoccupation pour l'environnement et l'implication envers les écolabels. La préoccupation pour l'environnement (PPE) est une variable centrale de la littérature portant sur l'explication des comportements proenvironnementaux (Giannelloni, 1998 ; Xiao et Dunlap, 2007). Elle semble primordiale à prendre en compte pour vérifier l'influence des croyances environnementales sur l'effet d'inclusion. En prolongation des travaux d'Irwin et Spira (1997), nous établissons l'hypothèse suivante : 
H2 : L'effet d'inclusion est plus fort chez les individus plus préoccupés par l'environnement que chez les individus moins préoccupés par l'environnement.

Vlosky, Ozanne et Fontenot (1999) proposent l'implication dans les écolabels comme indicateur de l'engagement du consommateur envers l'environnement. Pour des produits certifiés, ils mettent en évidence l'influence de l'implication envers la certification de produits en bois sur le consentement à payer. Dans notre recherche, l'implication a été définie comme un état de pertinence, d'attirance et d'intérêt envers un produit ou une cause sociale (Strazzieri, 1994). Un consommateur impliqué envers un écolabel devrait donc être moins sensible au nombre d'attributs environnementaux. Ainsi, nous émettons l'hypothèse suivante :

H3 : L'effet d'inclusion est plus fort chez les individus plus impliqués envers les écolabels que chez les individus moins impliqués envers les écolabels.

Divers facteurs sont susceptibles d'influencer l'effet d'inclusion, tels que la désirabilité sociale, l'absence de corrélation perçue entre les deux attributs environnementaux et l'attente envers la qualité du produit. La désirabilité sociale vise à chercher l'appui de ses pairs en donnant une réponse socialement acceptable, par exemple en signalant un engagement envers la cause environnementale. Beaucoup utilisée en marketing, elle constitue une motivation qui pourrait influencer l'effet d'inclusion. L'absence de corrélation naturelle perçue entre les deux attributs environnementaux rend l'effet d'inclusion non rationnel (Irwin et Spira, 1997). Nous prenons donc soin de vérifier les corrélations perçues - déclarées et mesurées- entre les attributs. L'attente envers la qualité (quality consciousness), c'est-à-dire le degré d'attention des consommateurs envers la qualité d'un produit, influence le comportement et notamment la valeur perçue des attributs de qualité (Steenkamp, 1989). Les individus davantage concernés par la qualité du produit sont mieux informés et, de ce fait, moins susceptibles de payer un surprix (Rao et Bergen, 1992 ; Rao et Monroe, 1996). Ils pourraient donc être moins sensibles au nombre des attributs environnementaux. Il est nécessaire de contrôler l'effet de ces trois variables dans ce travail.

Pour résumer, nous étudions l'influence du nombre d'attributs (1 vs. 2) et de leur nature (certifié $v s$.non certifié), de la préoccupation pour l'environnement et de l'implication envers les écolabels, ainsi que leurs interactions sur le poids des attributs environnementaux dans l'évaluation du produit par le consommateur. La Figure 1 présente le modèle testé et les relations analysées.

\section{(Insérer Figure 1)}

\section{MÉTHODE}


Deux expérimentations sont réalisées aux États-Unis pour tester les hypothèses, en manipulant le nombre d'attributs environnementaux (1 vs. 2) et leur nature (certifié vs. non-certifié). La première expérimentation teste l'effet d'inclusion pour des attributs certifiés (écolabel) ou noncertifiés (nom de marque) et l'effet modérateur de la PPE. Une seconde expérimentation teste si deux écolabels se font concurrence en montrant un effet d'inclusion. Pour ce faire, elle comporte uniquement des attributs certifiés, car le niveau d'engagement environnemental est étudié. Le stimulus comporte deux autres attributs neutres, en plus du prix et des attributs environnementaux, pour étudier l'effet d'inclusion en présence d'un nombre plus important d'attributs de produit. L'expérimentation 2 teste aussi les rôles modérateurs de PPE et de l'implication envers les écolabels et contrôle une série de variables explicatives (i.e. désirabilité sociale, corrélations perçues, attente envers la qualité).

\section{Stimulus}

Le stimulus choisi est un bureau en bois ; il a déjà été utilisé dans la littérature portant sur la prise de décision pour des produits avec attributs environnementaux (Ehrich et Irwin, 2005 ; Irwin et Baron, 2001). De plus, la gestion durable des forêts et de l'exploitation du bois est un point important de la démarche de développement durable. Des attributs spécifiques ont été créés pour les produits en bois ou dérivés (e.g. papier, carton), signalant une qualité intangible du produit (Veisten, 2007). Un exemple assez marquant est la présence quasi automatique des labels PEFC (Program for the Endorsement of Forest Certification ou Programme de Reconnaissance des Certifications Forestières) ou FSC (Forest Stewardship Council), garantissant une gestion durable des forêts, au dos des tickets de caisse.

\section{Plan et protocole expérimental}

Pour mettre en évidence l'effet d'inclusion, un plan intergroupe est utilisé pour tester la condition «nombre d'attributs environnementaux » (1 vs. 2), comme le conseillent Kahneman et Knetsch (1992b). Trois conditions sont créées : un groupe avec des options à évaluer ayant deux attributs environnementaux (condition A), et deux groupes avec des options à évaluer n'ayant que l'un ou l'autre attribut environnemental (conditions B et C). Pour rendre la tâche plus réaliste, un prix est attribué aux bureaux. Les trois attributs utilisés (le prix et les deux attributs environnementaux) comportent deux modalités chacun. Les modalités de prix ( $\$ 350$ ou $\$ 550$ ) sont reprises du travail d'Irwin et Baron (2001). Elles correspondent à des prix moyens du marché pour des bureaux en bois brut (e.g. 50\% des bureaux en bois proposés par Leguide.com sont dans cette gamme de prix) et restent les mêmes dans les deux expérimentations. Dans la condi- 
tion $\mathrm{A}$, les répondants ont 8 options à évaluer ( 3 attributs ayant chacun 2 modalités : le prix et 2 attributs environnementaux). Dans les conditions $\mathrm{B}$ et $\mathrm{C}$, les répondants ont 4 options à évaluer ( 2 attributs ayant chacun 2 modalités : le prix et 1 attribut environnemental; les Tableaux A1a et A1b -AnnexeA1- illustrent les options par condition). Certains auteurs montrent que l'effet d'ordre pourrait expliquer l'effet d'inclusion (Carson, 1997). Pour éviter cette influence, deux précautions sont prises : (1) la présentation aléatoire des options ; (2) la présentation des attributs aux répondants avant la tâche d'évaluation (Bateman et alii, 1997 ; Ehrich et Irwin, 2005). Le contexte d'évaluation est expliqué au répondant, ainsi que les attributs et la tâche à réaliser. Le protocole utilisé dans l'expérimentation 1 est détaillé en Annexe A1. Les répondants américains évaluent les options qui leur sont présentées puis répondent à une série de questions (PPE, familiarité du produit, âge, genre, anglais comme première langue).

\section{Évaluation globale des produits et calcul des poids des attributs}

La spécificité de ce travail est d'utiliser l'analyse conjointe, en présentant les différentes options de manière séparée (i.e. l'une après l'autre), alors qu'elles sont généralement présentées simultanément (Jolibert et Jourdan, 2006). Les répondants évaluent globalement les produits en donnant leur probabilité d'achat (PA) «Quelle est la probabilité que vous achetiez ce bureau ?», comprenant 7 échelons, de «pas du tout probable» à «tout à fait probable». Ce choix de mesure d'évaluation est motivé par les raisons suivantes : (1) la mesure de PA est largement employée en marketing pour l'évaluation de produit mais très peu dans les analyses de l'effet d'inclusion qui utilisent principalement des mesures monétaires (e.g. consentement à payer, Tableau 1) ; (2) l'analyse conjointe a été choisie pour réaliser une mesure indirecte du poids des attributs. En plus d'être une mesure plus réaliste, les deux autres intérêts de la méthode de l'analyse conjointe dans ce travail (par rapport à l'analyse contingente) est qu'elle permet d'obtenir le poids donné par chaque répondant pour chaque modalité d'attributs du produit ainsi que l'importance relative des poids des attributs (Le Gall-Ely et Robert-Demontrond, 2005) ; (3) la difficulté de la tâche peut exacerber l'effet d'inclusion (Fischhoff et alii, 1993). Pour éviter ce dernier problème, les options sont présentées séquentiellement de manière aléatoire, ce qui rend la tâche d'évaluation plus facile que dans le cas où toutes les options sont présentées simultanément.

Pour chaque répondant, les probabilités d'achat (PA) données pour chaque option sont régressées sur les modalités des attributs. Les coefficients de la régression pour les attributs sont utili- 
sés pour chaque individu ${ }^{1}$ (Ehrich et Irwin, 2005 ; Lefkoff-Hagius et Mason, 1993). Les modèles de régression utilisés pour chaque répondant sont les suivants :

(1) Condition $A: P A=b 0+b 1 *$ attribut $1+b 2 *$ attribut $2+b 3 *$ prix $+e_{P A}$

(2) Conditions $B(C): P A=b 0+b 1(2) * a t t r i b u t 1(2)+b 3 *$ prix $+e_{P A}$

dans lesquels les coefficients de régression $b 1$ et $b 2$ sont les poids des attributs environnementaux et $b 3$ est le poids du prix. Les poids moyens des attributs dans chaque condition sont calculés pour mener nos analyses : $M_{1}=\frac{1}{n} \sum_{\mathrm{i}=1}^{\mathrm{n}} b 1_{i} ; M_{2}=\frac{1}{n} \sum_{\mathrm{i}=1}^{\mathrm{n}} b 2_{i}$ et $M_{3}=\frac{1}{n} \sum_{\mathrm{i}=1}^{\mathrm{n}} b 3_{i}$. Le Tableau 2 présente les poids moyens des deux expérimentations.

\section{EXPÉRIMENTATION 1}

L'objectif de l'expérimentation 1 est de tester l'effet d'inclusion pour des attributs certifiés ou non-certifiés (H1), ainsi que le rôle modérateur de la PPE du consommateur sur cet effet d'inclusion (H2).

\section{Méthode de l'expérimentation 1}

L'expérimentation 1 est réalisée en laboratoire au sein d'une université américaine auprès d'étudiants de licence recevant un crédit pour leur participation. Elle emploie un attribut environnemental certifié (un écolabel) et un attribut non-certifié (un nom de marque), correspondant à la réalité du marché (Comas et Seifert, 2012). L'écolabel retenu pour cette expérimentation est le PEFC, label international pour les produits en bois, certifiant une gestion durable de la forêt. Le label est expliqué aux répondants avant la tâche d'évaluation (Annexe A1). Pour limiter les biais d'histoire, deux noms de marque fictifs sont créés (ProVerde et Beavara) et sont présentés aux répondants (Annexe A1 et Tableau A1a). 174 questionnaires sont reçus dont 1 est non exploitable (question de vérification non atteinte utilisée pour vérifier la lecture attentive). Sur les 173 questionnaires restants, 56 sont affectés en condition A ( 2 attributs), 58 en condition B (écolabel seul) et 59 en condition C (nom de marque seul). L'âge moyen des répondants est de 20 ans $(\sigma=1,81), 73,4 \%$ parlent l'anglais en première langue, 39,9\% sont des hommes et 6 personnes ont déjà vu l'écolabel (3,5\%). Les trois groupes ne sont pas statistiquement différents en termes de genre, d'âge et de familiarité au produit.

Xiao et Dunlap (2007) étudient la dimensionnalité de la PPE en reprenant un ensemble d'échelles de mesure utilisées dans la littérature mais ne peuvent conclure à

\footnotetext{
${ }^{1}$ Les modalités des attributs utilisent un codage orthogonal (-1 ou 1).
} 
l'unidimensionnalité du concept. La complexité d'approche nous amène à choisir une des mesures proposée par ces auteurs, appelée « importance de l'environnement ». Elle paraît intéressante pour sa simplicité de mesure ( 3 items, voir Annexe A3) et pour sa très bonne validité convergente $(0,95)$ avec le construit latent. Elle est donc choisie pour mesurer la PPE des individus. Une dimension résulte de l'analyse factorielle (valeur propre du facteur =2,23 avec un pourcentage de variance expliquée de 74,37\%; alpha de Cronbach =0,80) (Annexe A2). Un score moyen des trois items est calculé pour étudier l'influence de la PPE sur la valorisation des attributs. L'avantage de cette méthode, souvent utilisée lors d'expérimentations (e.g. Hong et Sternthal, 2010 ; White, MacDonnell et Dahl, 2011 ; Xu, Jiang et Dhar, 2013), est qu'elle permet de conserver des scores bruts.

\section{Résultats de l'expérimentation 1}

Dans un premier temps, le succès des manipulations expérimentales des attributs a été vérifié. Les répondants ayant évalué uniquement un bureau avec un écolabel ( $\left.\mathrm{M}_{\mathrm{PEFC}-\text { condB }}\right)$ perçoivent un produit avec le label PEFC comme étant effectivement meilleur pour l'environnement que ceux sans l'écolabel mais avec le nom de marque à évaluer ${ }^{2}\left(\mathrm{M}_{\mathrm{PEFC}-\text { condC }}\right)\left(\mathrm{M}_{\mathrm{PEFC}-\text { condB }}=5,37(\sigma=0,99)\right.$ vs. $\left.\mathrm{M}_{\mathrm{PEFC}-\text { condC }}=5,03(\sigma=0,93), \mathrm{t}(114)=1,95 \mathrm{p}=0,054\right)$. Les répondants perçoivent un produit avec le nom de la marque environnementale comme étant meilleur pour l'environnement qu'un produit avec le nom de marque non environnementale $\left(\mathrm{M}_{\text {envi. }}=4,79(\sigma=1,15) \mathrm{M}_{\text {non envi. }}=3,68\right.$ $\left.(\sigma=1,10), \mathrm{M}_{\text {diff envi }- \text { non envi }}=1,10(\sigma=1,45), \mathrm{t}(172)=10,04 \mathrm{p}<0,0001\right)$.

Les poids des attributs. Le Tableau 2 reprend les moyennes $\left(\mathrm{M}_{\mathrm{ij}}\right)$ des poids de régression pour

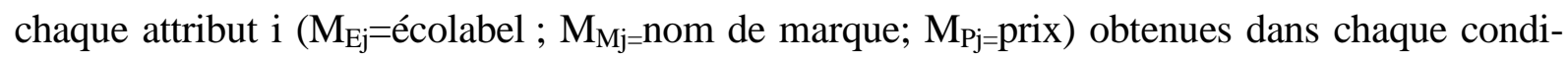
tion $\mathrm{j}\left(\mathrm{M}_{\mathrm{iA}}=2\right.$ attributs $; \mathrm{M}_{\mathrm{iB}}=$ écolabel ; $\mathrm{M}_{\mathrm{iC}}=$ nom de marque $)$.

\section{(Insérer le Tableau 2)}

Le Tableau 2 montre que le prix est l'attribut qui a le plus de poids. Ce travail portant sur la valorisation des attributs environnementaux, une comparaison est réalisée entre les poids attribués à l'écolabel certifié et au nom de marque non-certifié, entre les groupes ou au sein du groupe selon les conditions. Les individus en condition A ayant été exposés aux deux attributs environnementaux, la comparaison est faite intra-groupe $\left(\mathrm{M}_{\mathrm{EA}} v s . \mathrm{M}_{\mathrm{MA}}\right)$. Par contre, les individus en conditions $\mathrm{B}$ et $\mathrm{C}$ ayant été exposés à un seul attribut environnemental, la comparaison est réalisée intergroupes $\left(\mathrm{M}_{\mathrm{EB}}\right.$ vs. $\left.\mathrm{M}_{\mathrm{MC}}\right)$. En condition $\mathrm{A}$, la différence de moyenne entre les

\footnotetext{
${ }^{2}$ L'item de vérification de la manipulation de l'écolabel est mesuré sur une échelle allant de 1 à 7 (« Pensez-vous qu'un produit avec le label PEFC est vraiment meilleur pour l'environnement? »).
} 
poids de l'écolabel et du nom de marque est statistiquement significative $\left(\mathrm{M}_{\mathrm{EA}}-\mathrm{M}_{\mathrm{MA}}=0,29\right.$ $(\sigma=0,84), t(55)=2,63 \mathrm{p}=0,011)$. De même, le poids de l'écolabel $\left(\mathrm{M}_{\mathrm{EB}}\right)$ est plus grand que celui du nom de marque $\left(\mathrm{M}_{\mathrm{MC}}\right)\left(\mathrm{M}_{\mathrm{EB}}=0,66(\sigma=0,69)\right.$ vs. $\left.\mathrm{M}_{\mathrm{MC}}=0,36(\sigma=0,72), \mathrm{t}(115)=2,31, \mathrm{p}=0,023\right)$. L'attribut certifié a globalement plus de poids que l'attribut non certifié (Tableau 2). On note une différence statistiquement significative du genre sur la valorisation de l'écolabel, les femmes favorisant davantage l'écolabel que les hommes $\left(\mathrm{M}_{\mathrm{F}}=0,62(\sigma=0,67)\right.$ vs. $\mathrm{M}_{\mathrm{H}}=0,36$ $(\sigma=0,66), \mathrm{t}(112)=2,05 \mathrm{p}=0,043)$. Par contre, l'âge, la familiarité au produit et l'anglais comme $1^{\text {ére }}$ langue n'ont pas d'effet sur le poids des attributs environnementaux.

Influence du nombre d'attributs sur le poids des attributs. L'effet d'inclusion est testé en régressant le poids de l'attribut environnemental (écolabel ou nom de marque) sur la condition «nombre d'attributs » (1 vs. 2). Les moyennes des poids de chaque attribut dans chaque condition sont présentées dans le Tableau 2. Les résultats présentés à la Figure 2, sont en accord avec H1 et montrent clairement un effet d'inclusion. Le poids global des deux attributs présentés ensemble est inférieur au poids global des deux attributs présentés seuls sur le produit.

\section{(Insérer Figure 2)}

L'impact du nombre d'attributs environnementaux (1 vs. 2) sur le poids du prix est aussi testé en utilisant une ANOVA et deux contrastes orthogonaux : le contraste 1 compare la différence entre la condition $\mathrm{A}$ et les conditions $\mathrm{B}$ et $\mathrm{C}$, tandis que le contraste 2 compare la condition $\mathrm{B}$ avec la condition C. Les résultats (Tableau 2) indiquent que le poids du prix est plus important en présence d'un seul attribut environnemental $\left(\mathrm{M}_{\mathrm{PB}}\right.$ et $\left.\mathrm{M}_{\mathrm{PC}}\right)$ que lorsqu'il accompagne deux attributs environnementaux $\left(\mathrm{M}_{\mathrm{PA}}\right)\left(\mathrm{M}_{\mathrm{PA}}=0,95(\sigma=1,00)\right.$ vs. $\mathrm{M}_{\mathrm{PB}}=1,68(\sigma=0,85)$ vs. $\mathrm{M}_{\mathrm{PC}}=1,54$ $(\sigma=0,77), \quad F(2,170)=10,97 \mathrm{p}<0,0001 ;$ contraste $1: \mathrm{t}(170)=4,61 \mathrm{p}<0,0001 ;$ contraste 2 : $\mathrm{t}(170)=0,85 \mathrm{p}=0,396)$. Ce résultat a déjà été observé dans la littérature concernant la relation prix-qualité (Steenkamp, 1989 ; Tellis et Gaeth, 1990). Lorsque le prix est le seul attribut, il est plus important pour le consommateur que lorsqu'il est accompagné d'autres informations (Huber, Holbrook et Kahn, 1986). Dans l'expérimentation 2, nous vérifions si cet effet se maintient en présence d'un plus grand nombre d'attributs de produit dans chaque condition.

Influence de la préoccupation pour l'environnement (PPE) sur l'effet d'inclusion. Les tests de modération de la PPE sur l'effet d'inclusion sont réalisés au moyen d'une régression utilisant un terme d'interaction (Chumpitaz et Vanhamme, 2003; Spiller et alii, 2013). La variable modératrice est centrée autour de la moyenne et le facteur «nombre d'attributs» (1 vs. 2) utilise un codage orthogonal (-1/1), comme le recommandent Irwin et McClelland (2001) pour interpréter correctement les coefficients de régression avec la méthode du projecteur (spotlight analysis, 
Irwin et McClelland, 2001, 2003 ; Irwin, 2009 ; Spiller et alii, 2013). Cette technique utilise des statistiques de base de l'analyse de régression pour analyser l'effet simple d'une variable à un niveau particulier sur autre variable, continue ou catégorielle (Spiller et alii, 2013). Les résultats montrent que la PPE a un effet positif direct sur le poids des deux attributs environnementaux (Écolabel : $\mathrm{t}(112)=5,27 \mathrm{p}<0,0001$; Nom de marque : $\mathrm{t}(113)=2,27 \mathrm{p}=, 025)$. Par contre, la PPE n'a pas d'effet direct sur le poids du prix $(\mathrm{t}(171)=-0,09 \mathrm{p}=0,931)$ et il n'y a pas d'effet d'interaction statistiquement significatif entre la PPE et le nombre d'attributs sur le poids de l'écolabel $\left(\mathrm{t}(112)=-1,54 \mathrm{p}=0,1262 \mathrm{R}^{2}=0,23\right)$. Par contre, comme l'illustre la Figure 3, la PPE a un effet d'interaction significatif sur le poids du nom de marque $(t(113)=-2,54 \mathrm{p}=0,013$ $\left.\mathrm{R}^{2}=0,14\right)$. Pour analyser le sens de l'interaction, la méthode du projecteur est utilisée. On obtient un effet statistiquement significatif de la condition «nombre d'attributs » (1 vs. 2) avec une PPE élevée, mais un effet non significatif avec une PPE faible (Figure 3). Ce résultat pour l'attribut non-certifié est en accord avec l'hypothèse $\mathrm{H} 2$ : les individus plus préoccupés par la cause environnementale seront plus sujets à l'insensibilité au nombre d'attributs présents sur le produit.

\section{(Insérer Figure 3)}

Une légère corrélation négative statistiquement significative apparait entre les poids des attributs environnementaux dans la condition A ( 2 attributs) $\left(r_{p}=-0,269 p=0,045\right)$. Cette corrélation mesurée, au voisinage du seuil de 0,05, pourrait exprimer en partie l'insensibilité au nombre dans cette expérimentation avec la valorisation moins grande des attributs environnementaux dans la condition A (2 attributs). L'apparition de cette corrélation mesurée entre les deux attributs environnementaux dans la condition A est vérifiée dans l'expérimentation 2.

\section{EXPÉRIMENTATION 2}

L'objectif de l'expérimentation 2 est de tester l'effet d'inclusion, pour deux attributs certifiés (H1), et d'évaluer le rôle modérateur de deux caractéristiques environnementales individuelles (PPE et implication envers les écolabels) sur l'effet d'inclusion (H2 et H3).

L'expérimentation 1 ayant montré le poids plus important accordé à l'écolabel PEFC, l'expérimentation 2 est donc centrée sur deux attributs certifiés qui ont chacun deux modalités d'intensité de l'attribut, faible versus élevée (au lieu de présente vs. absente), pour tester si l'insensibilité au nombre d'attributs ( 1 vs. 2) se maintient. Dans le but de compléter les analyses de l'effet d'inclusion, un modérateur est aussi ajouté : l'implication envers les écolabels. Trois variables pouvant expliquer l'insensibilité au nombre d'attributs sont également analysées : la désirabilité sociale, le fait que la corrélation déclarée entre les deux attributs environnementaux 
soit forte et l'attente envers la qualité du produit. Enfin, pour dissimuler d'avantage l'objet de l'expérimentation et d'évaluer si l'effet d'inclusion se maintient avec plus d'attributs, deux attributs neutres (types de pied et de vis) sont ajoutés au plan expérimental. Ils ont trois intérêts : (1) camoufler l'objet de l'expérimentation, (2) vérifier l'effet du nombre d'attributs sur leurs poids, (3) maintenir un nombre constant d'options à évaluer entre les groupes.

\section{Méthode de l'expérimentation 2}

Un pré-test est réalisé pour choisir les deux attributs neutres et leurs deux niveaux, avec pour objectifs de sélectionner des attributs peu importants pour un bureau en bois et de vérifier la préférence peu marquée entre deux modalités des attributs (les résultats du pré-test sont détaillés en Annexe A4.1).

L'expérimentation 2 est réalisée en ligne sur un échantillon de convenance américain (les participants sont dédommagés $\$ 0,50$ pour leur participation). Elle compte deux attributs environnementaux certifiés (pourcentage de bois recyclé et empreinte carbone), choisis pour deux raisons. Premièrement, ils renvoient à la logique du développement durable environnemental indiquant une volonté globale de diminution de l'impact énergétique de l'entreprise. Deuxièmement, des attributs assez proches mais non-certifiés avaient été utilisés dans le travail de référence (Irwin et Spira, 1997) pour un autre type de produit (voiture). La sélection d'attributs semblables, mais certifiés dans un autre contexte, permet de vérifier la convergence des résultats obtenus et d'étudier si deux écolabels rivalisent et montrent un effet d'inclusion. Pour rendre les attributs crédibles, il est expliqué aux répondants que ces deux écolabels sont certifiés par l'EPC (Environmental Product Council), un organisme fictif tiers indépendant repris du travail de Luchs et alii (2010). En plus du prix (\$350 vs. \$550 comme dans l'expérimentation 1) et des deux attributs neutres, les attributs environnementaux suivants sont présentés aux répondants :

- Pourcentage de bois recyclé contenu dans le produit : $21 \%$ vs. 14\% de bois recyclé ;

- Empreinte carbone (engagement à réduire l'émission de gaz à effet de serre dans les 12 mois) : $10 \%$ vs. $25 \%$ d'émission de $\mathrm{CO} 2$ en moins.

Le protocole et la tâche d'évaluation se déroulent globalement comme en expérimentation 1 (Annexe $\mathrm{A} 1$ et Tableau $\mathrm{A} 1 \mathrm{~b}$ ), à quelques détails près. Les attributs neutres sont présentés et ajoutés de manière aléatoire afin de maintenir huit options à évaluer dans les trois conditions. Des questions supplémentaires apparaissent à l'issue de la tâche d'évaluation : l'implication envers les écolabels, la désirabilité sociale, l'attente envers la qualité, la corrélation déclarée entre les deux attributs environnementaux, et des questions sociodémographiques (statut mari- 
tal, revenu, enfants). 188 questionnaires sont collectés dont 19 sont non exploitables : 11 n'ont pas bien répondu à la question de vérification et 8 répondants sont non anglophones, ce qui peut biaiser la compréhension des questions. Sur les 169 questionnaires restants, 56 sont affectés en condition A (2 écolabels), 58 en condition B (écolabel émission de $\mathrm{CO} 2$ seul) et 55 en condition C (écolabel matière recyclée seul). L'âge moyen des répondants est de 32,52 ans $(\sigma=12,47)$, $40,2 \%$ sont des hommes, $61,5 \%$ ont un revenu du foyer inférieur à $\$ 50$ 000, 63,3\% n'ont pas d'enfant $\left(M_{\text {enfants }}=0,76(\sigma=1,23)\right)$ et $41,4 \%$ vivent en couple. Les trois groupes sont comparables en termes de genre, statut marital, revenu, moyenne d'âge, de familiarité à l'égard du produit et du nombre d'enfants.

Les propriétés statistiques des échelles d'intervalle sont testées et la validité discriminante et convergente des échelles est vérifiée par des analyses factorielles exploratoires et confirmatoires (Annexe A2 et Tableau A2). La mesure de PPE, utilisée en expérimentation 1, est reprise pour l'expérimentation 2 (Annexe A3). Elle présente également une dimension à l'issue de l'analyse factorielle et une bonne fiabilité et cohérence interne (alpha de Cronbach=0,91; $\rho$ de Jöres$\left.\operatorname{kog}=0,92 ; \rho_{\mathrm{vc}}=0,79\right)$ (Annexe A2). L'implication envers l'écolabel est mesurée à l'aide des 4 premiers items de l'échelle d'implication de Strazzieri (1994) pour des raisons de parcimonie. Ils sont transposés aux écolabels par deux experts francophones. Les items ont ensuite été traduits en anglais puis rétro-traduits en français par deux couples de traducteurs différents. L'échelle française d'implication envers les écolabels est présentée en Annexe A3. Une dimension résulte de l'analyse factorielle (81,26\% de variance expliquée par un facteur). L'échelle est fiable et valide (alpha de Cronbach=0,92; $\rho$ de Jöreskog=0,92; $\rho_{\mathrm{vc}}=0,75$ ) (Annexe A2). Un score moyen est calculé pour ces deux échelles de mesure. Les échelles mesurant l'attente envers la qualité, la désirabilité sociale et les corrélations perçues sont présentées en Annexe A3.

\section{Résultats de l'expérimentation 2}

Le succès des manipulations expérimentales des attributs est vérifié. Un produit avec un écolabel de réduction élevée d'émission de $\mathrm{CO} 2\left(\mathrm{M}_{\mathrm{CO}_{2}}\right)$ et un écolabel de pourcentage élevé de contenu recyclé $\left(\mathrm{M}_{\mathrm{recy}+}\right)$ est perçu par les répondants comme étant meilleur pour l'environnement qu'un produit avec les mêmes écolabels de modalités plus faibles $\left(\mathrm{M}_{\mathrm{CO}_{2}-}\right.$ et $\left.\mathrm{M}_{\mathrm{recy}-}\right)\left(\mathrm{M}_{\mathrm{CO} 2+}=5,49\right.$ $(\sigma=1,23)$ vs. $\mathrm{M}_{\mathrm{CO} 2}=4,91(\sigma=1,09), \mathrm{M}_{\text {diff CO2+ - CO2- }}=0,59(\sigma=1,23), \mathrm{t}(168)=7,55 \mathrm{p}<0,0001 ; \mathrm{M}_{\text {re- }}$ ${ }_{\text {cy+ }}=5,25(\sigma=1,14)$ vs. $\mathrm{M}_{\text {recy- }}=4,75 \quad(\sigma=1,05), \mathrm{M}_{\text {diff }}$ recy+ - recy-=0,50 $(\sigma=0,77), \mathrm{t}(168)=8,55$ $\mathrm{p}<0,0001$ ). La neutralité des attributs (type de vis et type de pieds) est également vérifiée, comme dans le pré-test : l'importance des deux attributs est moyenne et la préférence est peu 
marquée entre les deux modalités des deux attributs (Annexe A4.2).

Les poids des attributs. Le Tableau 2 reprend les moyennes $\left(\mathrm{M}_{\mathrm{ij}}\right)$ des poids de régression pour chaque attribut i $\left(\mathrm{M}_{\mathrm{Cj}}=\right.$ réduction élevée d'émission de $\mathrm{C} 02 ; \mathrm{M}_{\mathrm{Rj}}=\%$ élevé de matière recyclée nom de marque; $\mathrm{M}_{\mathrm{Pj}}=$ prix $)$ obtenues dans chaque condition $\mathrm{j}\left(\mathrm{M}_{\mathrm{iA}}=2\right.$ écolabels ; $\mathrm{M}_{\mathrm{iB}}=\mathrm{e}$ mission de $\mathrm{C} 02 ; \mathrm{M}_{\mathrm{iC}}=$ matière recyclée). De nouveau, le prix est l'attribut qui a le plus de poids. La comparaison entre les poids des deux écolabels est réalisée comme dans l'expérimentation 1. En condition A (2 écolabels), la différence de moyenne entre les poids des deux écolabels n'est pas statistiquement significative $\left(\mathrm{M}_{\mathrm{CA}}-\mathrm{M}_{\mathrm{RA}}=-0,005(\sigma=0,58), \mathrm{t}(55)=-0,06 \mathrm{p}=0,954\right)$. De même, le poids des deux écolabels n'est pas statistiquement significativement différent entre les conditions $B$ et $C\left(M_{C B}=0,53(\sigma=0,64) v s . M_{R C}=0,52(\sigma=0,565), t(111)=0,135 p=0,893\right)$. Les poids des deux écolabels sont globalement comparables. Une différence statistiquement significative du nombre d'enfants apparait sur le poids de l'écolabel $\mathrm{CO} 2+$; plus les répondants ont un nombre d'enfants élevé, plus ils valorisent ce label $(\mathrm{t}(112)=2,52, \mathrm{p}=0,013)$. Il n'y a pas d'effet statistiquement significatif de l'âge, du genre, de la familiarité envers le produit, du revenu, du statut marital sur le poids des deux attributs environnementaux, ni du nombre d'enfants sur le poids de l'écolabel matière recyclée (recy+).

Influence du nombre d'attributs sur le poids des attributs environnementaux. L'effet d'inclusion est testé en régressant la condition «nombre d'attributs » (1 vs. 2) sur le poids de l'attribut environnemental (émission de $\mathrm{CO} 2$ et matière recyclée). Le Tableau 2 reprend les moyennes des poids dans chaque condition. Un effet significatif direct est mis en valeur pour les deux attributs environnementaux. Le poids global des attributs environnementaux présentés ensemble est inférieur au poids global des ces deux attributs présentés seuls sur le produit. Ces résultats, en accord avec H1, indiquent clairement un effet d'inclusion (Figure 4).

\section{(Insérer Figure 4)}

L'impact du nombre d'attributs sur le poids du prix est aussi testé, en utilisant la même méthode que pour l'expérimentation 1. Les résultats indiquent que le poids du prix n'est pas différent dans les trois groupes $\left(\mathrm{M}_{\mathrm{PA}}=0,95(\sigma=0,93)\right.$ vs. $\mathrm{M}_{\mathrm{PB}}=1,01 \quad(\sigma=0,94)$ vs. $\mathrm{M}_{\mathrm{PC}}=1,16 \quad(\sigma=1,15)$ $\mathrm{F}(2,166)=0,65 \mathrm{p}=0,524$ ) et n'est pas influencé par le nombre d'attributs (au minimum trois attributs en plus du prix contre un en expérimentation 1), comme le suggèrent les travaux antérieurs (Huber et McCann, 1982).

Influence de la PPE sur l'effet d'inclusion. Les tests de modération de la PPE et de l'implication envers les écolabels sur l'effet d'inclusion sont réalisés en suivant la même méthode que pour 
l'expérimentation 1 (i.e. régression avec un terme d'interaction). La PPE a un effet positif direct sur le poids des deux attributs environnementaux (émission de $\mathrm{CO} 2: \mathrm{t}(112)=4,66 \mathrm{p}<0,001$; matière recyclée: $\mathrm{t}(109)=2,23 \mathrm{p}=0,0280$ ). Les individus plus (moins) préoccupés par l'environnement donneront un poids plus (moins) grand aux écolabels $\mathrm{CO} 2+$ et recy+. La PPE a un effet d'interaction statistiquement significatif sur le poids de $\mathrm{CO} 2+(\mathrm{t}(112)=-2,80 \mathrm{p}=0,006$ $\left.\mathrm{R}^{2}=0,25\right)$ mais pas sur le poids de matière recyclée (recy+) $\left(\mathrm{t}(109)=-1,60 \mathrm{p}=0,113 \mathrm{R}^{2}=0,09\right)$. Pour analyser le sens de l'interaction, la méthode du projecteur est de nouveau utilisée. Un effet statistiquement significatif de la condition «nombre d'attributs » est obtenu lorsqu'on projette en PPE élevée. Par contre, pour un PPE faible, l'effet de la condition devient non significatif (Figure 5). Ce résultat pour le label CO2+ est en conformité avec l'hypothèse $\mathrm{H} 2$ : les individus plus préoccupés par l'environnement seront davantage sujets à l'insensibilité au nombre d'attributs présents sur le produit.

\section{(Insérer Figure 5)}

Influence de l'implication envers les écolabels sur l'effet d'inclusion. L'implication envers les écolabels a un effet positif direct sur le poids des deux attributs environnementaux (émission de $\mathrm{CO} 2: \mathrm{t}(112)=4,23 \mathrm{p}>0,0001$; matière recyclée : $\mathrm{t}(109)=3,59 \mathrm{p}=0,001)$. Ainsi, plus les individus sont impliqués envers les écolabels, plus le poids alloué aux écolabels $\mathrm{CO} 2+$ et recy+ sera grand. L'implication envers les écolabels a aussi un effet d'interaction statistiquement significatif sur le poids du label $\mathrm{CO} 2+\left(\mathrm{t}(112)=-2,31 \mathrm{p}=0,023 \mathrm{R}^{2}=0,17\right)$ mais un effet non statistiquement significatif sur le poids du label matière recyclée (recy+) $\left(\mathrm{t}(109)=-1,67 \mathrm{p}=0,097 \mathrm{R}^{2}=0,14\right)$. Les résultats des tests utilisant la méthode du projecteur mettent en évidence qu'en état d'implication élevée, l'effet d'inclusion a une influence statistiquement significative sur le poids de $\mathrm{CO} 2+$ tandis qu'il ne l'a pas en état d'implication faible. La Figure 6 illustre l'effet d'interaction sur le poids de $\mathrm{CO} 2+$. Ce résultat pour le label $\mathrm{CO} 2+$ est en accord avec l'hypothèse $\mathrm{H} 3$ : les individus qui sont plus impliqués envers les écolabels seront moins sensibles au nombre d'attributs présents sur le produit.

\section{(Insérer Figure 6)}

Cette modération des croyances environnementales sur l'effet d'inclusion apparait particulièrement marquée pour l'attribut «émission de $\mathrm{CO} 2$ », indiquant qu'il s'agit d'un attribut plus prototypique ou représentatif de la catégorie environnementale (Cohen et Basu, 1987). Ce résultat confirme ceux d'Irwin et Spira (1997). Par contre, ni la PPE (t(167)=0,03 p=0,977), ni l'implication envers les écolabels $(\mathrm{t}(167)=1,69 \mathrm{p}=0,093)$ n'ont un effet direct statistiquement significatif sur le poids du prix. Par ailleurs, aucun effet d'interaction statistiquement significatif 
entre le nombre d'attributs et une série de variables (la désirabilité sociale, l'attente envers la qualité et les corrélations perçues -déclarées et mesurées- entre les attributs environnementaux sur les poids des attributs) n'est mis en évidence (le détail des analyses est disponible en Annexe A4.3).

\section{DISCUSSION}

Dans cette recherche, l'effet d'inclusion est mis en évidence dans les deux expérimentations. Les individus ont tendance à sous-estimer un attribut environnemental si le produit en comprend un autre. Autrement dit, le poids donné à un attribut sera plus faible si un autre attribut est présent sur le même produit. L'expérimentation 1 montre que l'effet d'inclusion apparait pour les attributs certifiés et non certifiés. Dans l'expérimentation 2, l'insensibilité au nombre apparait pour des attributs certifiés ayant des modalités «faible » ou «élevée » (e.g. \% de matière recyclée, réduction d'émission de C02) et n'est expliquée ni par la désirabilité sociale, ni par les corrélations perçues entre les attributs, ni par l'attente envers la qualité des produits. Les résultats mettent aussi en avant le fait que les individus ne sous-estiment pas toujours un attribut environnemental inclus dans une combinaison d'attributs. Ainsi, la PPE va modérer l'insensibilité au nombre d'attributs (expérimentations 1 et 2). Les individus plus impliqués envers les écolabels seront plus sujets à l'effet d'inclusion (expérimentation 2). Ce dernier point constitue un résultat clé de ce travail car il permet de mieux comprendre les conditions d'apparition de l'effet d'inclusion dans un contexte commercial.

D’un point de vue théorique, les résultats obtenus sur des attributs environnementaux certifiés sont conformes aux travaux généraux antérieurs qui avancent trois explications-clés à cette insensibilité au nombre d'attributs présents sur le produit : (1) les individus ont tendance à signaler leur engagement pour acheter de la satisfaction morale ; (2) les attributs environnementaux peuvent renvoyer à des valeurs protégées ; (3) un attribut environnemental peut suffire à catégoriser un produit comme pro-environnemental. Ces propositions conduisent à la prédiction que, quand les évaluations des individus sont basées exclusivement sur leur perception de l'aspect environnemental d'un produit, ajouter un attribut environnemental peut en fait diminuer, plutôt qu'augmenter, le poids de l'attribut déjà présent sur le produit. Nos résultats montrent que le poids d'un attribut diminue si un autre est présent, le poids global de deux attributs pouvant même être inférieur au poids d'un attribut environnemental seul. Ces résultats ont une implication importante dans l'estimation des préférences pour des produits ayant au moins deux attributs non marchands. Réaliser des études distinctes pour mesurer le poids relatif alloué à chacun 
des attributs conduirait à un poids global prédit bien supérieur à leur poids réel. L'effet d'inclusion semble donc constituer une explication théorique à prendre en compte en marketing pour l'évaluation de produits ayant des caractéristiques non marchandes dans des contextes variés (e.g. environnemental, santé, social). Par exemple, associer un attribut social à un attribut environnemental sur un produit, comme une double labellisation biologique et équitable, pourrait se traduire par un effet négatif pour l'évaluation du produit par le consommateur. De même, en marketing sociétal, la multiplication d'arguments de prévention pour une campagne de santé pourrait s'avérer contre-productive.

Nos résultats sont aussi à rapprocher des travaux concernant un autre biais systématique : le biais de moyenne. Ce dernier, mis en évidence dans le domaine alimentaire, conduit à une évaluation calorique moins élevée d'un plat contenant un aliment gras et un aliment sain que l'évaluation d'un plat contenant uniquement un aliment gras (Chernev et Gal, 2010). La transposition de cet effet à notre étude impliquerait que les individus engagés dans la cause environnementale utiliseraient une règle non pas additive mais de moyenne lors de l'évaluation d'un produit avec plusieurs attributs environnementaux. Combiner deux attributs pourrait ainsi amener à de moins bonnes évaluations par rapport à un seul attribut.

Les expérimentations ont aussi mis en évidence l'influence de critères sociodémographiques (nombre d'enfants et genre) sur le poids des attributs environnementaux. Ces résultats confirment ceux d'autres recherches qui ont souligné le lien entre ces critères et des comportements pro-environnementaux. Dietz, Kalof, et Stern (2002) montrent que les femmes sont plus sensibles à l'environnement que les hommes; Brécard et alii (2009) mettent en évidence que le profil type d'un acheteur de poisson éco-labélisé est une femme jeune et éduquée ; Whitmarsh et O'Neill (2010) soulignent l'effet positif du nombre d'enfants sur les comportements proenvironnementaux.

Au niveau pratique, cette recherche, réalisée sur des attributs environnementaux, a des implications managériales et de politique publique importantes. Malgré l'augmentation du nombre d'attributs environnementaux apposés, la consommation des produits de cette catégorie ne crôtt pas de manière proportionnelle (Luchs et alii, 2010). Notre recherche met effectivement en évidence une limite potentielle à la multiplication du nombre d'attributs environnementaux sur les produits. Trois conséquences clés peuvent être alors exploitées par les chefs de produits : (1) les individus ont tendance à catégoriser les produits possédant au moins un attribut comme proenvironnementaux ; (2) les individus perçoivent un produit combinant plusieurs attributs environnementaux comme étant peu différent d'un produit ayant un seul attribut environnemental et 
(3) ces conclusions s'appliquent particulièrement aux individus engagés envers l'environnement. Ce travail trace donc les limites d'un positionnement «produit proenvironnemental » et de la démarche marketing associée. Les écolabels ont plus de poids mais l'accumulation des écolabels ou d'autres signaux non-certifiés n'apparait pas comme une stratégie efficace de positionnement.

Si la sensibilité au prix semble diminuer avec un nombre plus élevé d'attributs environnementaux (expérimentation 1), cet effet n'est pas retrouvé pour un produit ayant un nombre plus important d'attributs (expérimentation 2). Le lien entre positionnement pro-environnemental et profit réalisé n'est donc pas direct, comme l'ont souligné d'autres études antérieures (Loureiro, McCluskey et Mittelhammer, 2002; Sedjo et Swallow, 2002). Les résultats de notre travail incitent plutôt les manageurs à ajouter un label certifié à un produit ayant un nom de marque existant, plutôt que de créer un nom de marque spécifique, permettant d'écouler le produit avec ou sans label à des prix différents. La remise en question du cumul des attributs environnementaux touche aussi la publicité pour ces produits, puisque une communication sur deux attributs serait moins valorisée par les consommateurs que deux communications sur un attribut.

\section{Limites et perspectives}

Même s'il a abouti à des résultats cohérents et conformes à ceux d'études antérieures, ce travail n'a pas couvert tous les aspects du problème de l'évaluation des attributs environnementaux et présente inévitablement des limites. Nous avons privilégié la validité interne de notre étude, entrainant des répercussions possibles sur la validité externe de nos résultats. Premièrement, les caractéristiques déclaratives (mesure et protocole) de notre expérimentation peuvent faire apparaitre un biais hypothétique (Miller et alii, 2011), conduisant à des valeurs données par les répondants plus hautes que celles fournies en conditions réelles. Nous avons eu recours à une mesure de préférence déclarée et nous avons expliqué les attributs avant la tâche d'évaluation, afin d'éviter les effets d'ordre et de s'assurer que les attributs utilisés étaient bien compris. Cette démarche peut induire une surpondération factice des poids des attributs et n'est pas nécessairement représentative de la réalité du marché. Deuxièmement, les choix du produit (un bureau en bois) et de l'échantillon d'étudiants peuvent être questionnés et mériteraient une réplication basée sur d'autres consommateurs afin de vérifier la validité des résultats. Troisièmement, nous avons adopté, dans l'expérimentation 1, deux attributs environnementaux dont la notoriété était faible (marque fictive et label peu connu), pour éviter la domination d'un attribut et les biais d'histoire. La faible notoriété déclarée pour l'écolabel n'a pas empêché les individus de le pren- 
dre en considération dans leur prise de décision en lui affectant un poids relativement important. Toutefois, utiliser des attributs peu connus ( $v s$. très connus) pourrait avoir un impact sur l'apparition de l'effet d'inclusion. La faible notoriété pourrait effectivement limiter la généralisation des résultats obtenus et cet effet devrait être étudié. Pour confirmer la prédiction de ces comportements, une réplication en condition plus incitative (i.e. achat possible à effectuer) serait nécessaire (Allenby et alii, 2005). Les conséquences en situation réelle de l'effet d'inclusion sur le choix des consommateurs pourraient ainsi être étudiées. Enfin, l'évaluation de plusieurs attributs environnementaux sujette à une insensibilité de grandeur pour les consommateurs engagés envers la cause, ne veut pas dire que le produit avec le plus d'attributs n'est pas choisi. Une étude portant sur le choix des consommateurs serait nécessaire pour compléter la compréhension du phénomène.

Les résultats de ce travail démontrent le besoin de sélectionner soigneusement les attributs environnementaux. Pour cette raison, Bougherara et Piguet (2008) indiquent que l'écolabel peut se révéler inefficace, à cause du coût de recherche d'information élevé qu'il introduit, surtout si le produit est acheté fréquemment. Ils conseillent de choisir les écolabels selon le type de produit, en distinguant les produits achetés fréquemment ou plus rarement, ces derniers étant plus adaptés à la présence d'un écolabel. Le besoin de sélectionner l'attribut environnemental selon le type de produit est mis en évidence dans le travail de Luchs et alii (2010) qui démontrent qu'un attribut environnemental (associé à plus de douceur) sera un atout pour un savon de bébé mais pas pour un savon de voiture. Les caractéristiques du producteur et la crédibilité perçue de son engagement sont aussi des variables influençant l'évaluation des produits comportant des critères environnementaux (e.g. le capital de marque et son effet négatif sur la valeur d'un attribut environnemental ; Larceneux, Benoit-Moreau et Renaudin, 2011). Ces résultats, ainsi que ceux de notre étude, ouvrent de futures voies de recherche sur les implications de l'effet d'inclusion dans des contextes variés. 


\section{ANNEXE A1 - PROTOCOLE EXPÉRIMENTAL}

\section{Exemple de protocole utilisé (expérimentation 1)}

«Imaginez que vous allez dans un grand magasin de mobilier pour acheter un nouveau bureau en bois. Cet achat est très important pour vous car vous comptez passer beaucoup de temps à votre bureau. Pendant que vous êtes dans l'allée du magasin considérant les nombreuses options offertes par le magasin, vous remarquez que tous les bureaux présentent trois [ou deux] informations (la taille, la couleur et tous les autres éléments sont par ailleurs similaires).

- Le nom de marque du bureau: Beavara (une marque de mobilier populaire et assez commune) ou Proverde (une marque de mobilier pro-environnementale impliquée de différentes façons dans la réduction de l'impact environnemental de sa production).

- Un écolabel : Le Programme de Reconnaissance des Certifications Forestières (PEFC) est une organisation internationale à but non lucratif, non gouvernementale dédiée à la promotion de la gestion durable des forêts grâce à la certification par un tiers indépendant. Il a pour objectif, tout au long de la chaîne d'approvisionnement forestier, de promouvoir les bonnes pratiques dans la forêt et de veiller à ce que les produits de bois brut ou dérivés soient fabriqués dans le respect des normes écologiques, sociales et éthiques.

\section{- Le Prix : $\mathbf{3 5 0}$ \$ ou $\mathbf{5 5 0} \$$}

Maintenant il est temps de choisir votre bureau. Imaginez que vous voyez 8 [ou 4] bureaux dans le magasin. Tous les bureaux sont dans votre budget (i.e. ils sont compris dans la fourchette de prix que vous envisagez de dépenser). Rappelez-vous que les bureaux varient selon 3 [ou 2] critères : le nom de la marque et [ou] l'écolabel, le prix. Supposez que les bureaux ne sont différents les uns des autres en aucune manière en dehors de ces critères. Nous aimerions maintenant que vous nous disiez à quel point vous êtes susceptible d'acheter chacun des bureaux. S'il vous plaît, lisez les informations de chaque bureau attentivement. Ils ne sont présentés dans aucun ordre particulier ».

\section{Illustration des options de chaque condition (expérimentations 1 et 2)}

Le répondant est affecté à une des trois conditions du Tableau A1 $(\mathrm{a}=$ expérimentation $1 / \mathrm{b}=$ expérimentation 2). Les options sont présentées aux répondants l'une après l'autre de manière aléatoire, à chaque fois il est demandé la probabilité d'achat sur une échelle à 7 échelons («pas du tout probable » à « tout à fait probable »).

\section{(Insérer Tableaux A1a et A1b)}




\section{ANNEXE A2 - VALIDITÉ DES ÉCHELLES DE MESURE}

\section{Expérimentation 1}

Préoccupation pour l'environnement. L'échelle composée de trois items, repris de Xiao et Dunlap (2007), présente une structure unidimensionnelle (ACP avec communauté minimum observée de 0,634, une valeur propre du facteur=2,23 avec un pourcentage de variance expliquée de 74,367\%, un $\mathrm{KMO}=0,687$ et le test de sphéricité de Bartlett $\chi^{2}=208,29 \mathrm{dl}=3$ p $<0,001$ ) et une bonne fiabilité (alpha de Cronbach=0,80).

\section{Expérimentation 2}

\section{(Insérer Tableau A2)}

Préoccupation pour l'environnement. L'échelle composée de trois items repris de Xiao et Dunlap (2007) présente une structure unidimensionnelle (ACP avec communauté minimum observée de 0,800, une valeur propre du facteur $=2,57$ avec un pourcentage de variance expliquée de 85,53\%, un $\mathrm{KMO}=0,738$ et le test de sphéricité de Bartlett $\chi^{2}=371,65 \mathrm{dl}=3 \mathrm{p}<0,001$ ) et une bonne fiabilité (alpha de Cronbach=0,91; $\rho$ de Jöreskog=0,92) et validité convergente $\left(\rho_{\mathrm{vc}}=0,79\right)$.

Implication envers les écolabels. L'échelle composée de 4 items adaptée de Strazzieri (1994) présente une structure unidimensionnelle (ACP avec communauté minimum observée de 0,656, une valeur propre du facteur=3,25 avec un pourcentage de variance expliquée de $81,26 \%$, un $\mathrm{KMO}=0,82$ et le test de sphéricité de Bartlett $\left.\chi^{2}=562,52 \mathrm{dl}=4 \mathrm{p}<0,001\right)$ et une bonne fiabilité (alpha de Cronbach=0,94; $\rho$ de Jöreskog $=0,92)$ et bonne validité convergente $\left(\rho_{\mathrm{vc}}=0,75\right)$.

Attente envers la qualité. L'échelle est composée de trois items, adaptée de Rao et Bergen (1992), présente une structure unidimensionnelle (ACP avec communauté minimum observée de 0,539 , une valeur propre du facteur $=1,81$ avec un pourcentage de variance expliquée de $60,23 \%$, un $\mathrm{KMO}=0,892$ et le test de sphéricité de Bartlett $\left.\chi^{2}=76,47 \mathrm{dl}=3 \mathrm{p}<0,001\right)$ et une fiabilité passable, inférieure au seuil de 0,7 recommandé par Nunnally (1978) (alpha de Cronbach $=0,65, \rho$ de Jöreskog $=0,68)$ et validité convergente médiocre $\left(\rho_{\mathrm{vc}}=0,42\right)$. La cohérence interne présente une faiblesse. L'échelle, ayant déjà été utilisée dans la littérature avec des propriétés statistiques équivalentes (Rao et Bergen, 1992), est toutefois conservée en variable de contrôle.

Comme l'illustre le Tableau A2, la validité discriminante des trois échelles est bonne. Le $\rho_{\mathrm{vc}}$ de chaque échelle est supérieur aux carrés des corrélations $\left(\phi^{2}\right)\left(\mathrm{PPE}: \rho_{\mathrm{vc}}=0,79>0,11\right.$ et 0,$51 ; \mathrm{Im}$ - 
plication envers les écolabels : $\rho_{\mathrm{vc}}=0,75>0,07$ et 0,51 ; Attente envers la qualité $=0,42>0,07$ et $0,11)$.

Désirabilité sociale. La tendance à répondre d'une manière socialement désirable a été mesurée à l'aide de la forme abrégée de l'échelle de Marlowe-Crowne de Reynolds (1982). L'échelle est composée de 13 comportements (cinq formulés positivement et huit négativement) (Annexe A3). Les modalités de réponses de l'échelle sont «vrai » ou «faux ». Un score final élevé sur l'échelle (variant de 0 à 13) indique la tendance du sujet à se présenter d'une manière socialement favorable. Les caractéristiques de l'échelle obtenues (un facteur par le test de Cattell et le test de Kuder Richardson-20=0,71) sont cohérentes avec les études antérieures (e.g. Reynolds, 1982). 


\section{ANNEXE A3 - LES ÉCHELLES DE MESURE UTILISÉES}

\section{Échelles de préoccupation pour l'environnement, reprise de Xiao et Dunlap (2007)*}

1 Pensez-vous que les problèmes environnementaux sont importants ?

2 Les problèmes environnementaux sont-ils une préoccupation personnelle ?

3 Pensez-vous que les questions environnementales sont sérieuses ?

\section{Échelle d'implication envers les écolabels, adaptée de Strazzieri (1994)*}

1 Un écolabel est un attribut de produit qui compte vraiment pour moi

2 Un écolabel est un attribut de produit auquel j'accorde une importance particulière

3 J'aime particulièrement parler d'écolabels

4 On peut dire que l'écolabel est un attribut de produit qui m'intéresse

\section{Échelle d'attente envers la qualité, adaptée de Rao et Bergen (1992)*}

1 Je pense qu'acheter des produits de haute qualité est essentiel

2 Je subirais une perte importante d'argent, si la qualité de ce produit était faible

3 En général, je m'assure que les produits et services qui me sont fournis sont d'un niveau de qualité acceptable

*Les réponses comportent 7 échelons de «pas du tout d'accord » à « tout à fait d'accord».

\section{Échelle de Désirabilité Sociale réduite à 13 items, reprise de Reynolds (1982)***}

1. Il est parfois difficile pour moi d'avancer sur mon travail si je ne suis pas encouragé(e). F

2. Je me sens parfois irrité(e) lorsque je n'obtiens pas ce que je veux. F

3. À quelques occasions, j'ai renoncé à faire quelque chose parce que je ne m'en sentais pas capable. F

4. Il y a eu des moments où j'ai eu envie de me rebeller contre les personnes en position d'autorité, même si je savais qu'elles avaient raison. $F$

5. Peu importe à qui je parle, je suis toujours à l'écoute. V

6. Il y a eu des moments où j'ai profité de quelqu'un. $F$

7. Je suis toujours prêt(e) à admettre quand je fais une erreur. V

8. J'essaie parfois de me venger plutôt que de pardonner et d'oublier. $\mathrm{F}$

9. Je suis toujours courtois(e), même avec des personnes qui sont désagréables. V

10. Je n'ai jamais été agacé(e) quand les gens ont exprimé des idées très différentes des miennes. V

11. Il y a eu des moments où j'ai été assez jaloux(se) de la bonne fortune d'autrui. $\mathrm{F}$

12. Je suis parfois agacé(e) par des personnes qui me demandent des faveurs. F

13. Je n'ai jamais dit de manière délibérée quelque chose qui blesse les sentiments de quelqu'un d'autre. $\mathrm{V}$

**Note $: \mathrm{V}=$ Vrai / F=Faux ; correspondent aux réponses attendues montrant un comportement de désirabilité sociale 
Corrélations perçues entre les attributs environnementaux, reprises de Irwin et Spira (1997)*** «Émission de $\mathrm{CO} 2$ - $\quad$ Si on vous disait qu' un produit avait de faibles émissions de $\mathrm{CO} 2$, alors le Matière recyclée » produit aurait probablement un important contenu de matière recyclée «Matière recyclée - $\quad$ Si on vous disait qu'un produit contenait beaucoup de matière recyclée, le Émission deC02» produit aurait probablement de faibles émissions de $\mathrm{CO} 2$ ***Les réponses comportent 9 échelons de «je ne le penserais jamais » $\grave{a} \ll j e ~ l e ~ p e n s e r a i s ~ t o u j o u r s »$. 


\section{ANNEXE A4 - EXPÉRIMENTATION 2}

\section{A4.1. Pré-test : sélection de deux attributs neutres}

Le pré-test est effectué en ligne sur un échantillon de convenance de 35 individus américains de moyenne d'âge de 35 ans $(\sigma=11)$, composé de $63 \%$ de femmes. Différents attributs d'un bureau en bois sont présentés aux répondants : essence de bois, type de vis, type de pieds, couleur, nombre de tiroirs et hauteur du bureau.

Pour mesurer leur importance, une échelle à 7 échelons («pas du tout important » à «tout à fait important ») est utilisée pour chacun des attributs. Les résultats indiquent que les types de vis et de pieds du bureau sont perçus comme des attributs relativement neutres. Le test $t$ indique que les moyennes des deux attributs ne sont pas significativement différentes de $3\left(M_{v i s}=2,71\right.$ $\left.(\sigma=1,73), \mathrm{t}(34)=-0,98 \mathrm{p}=0,334 ; \mathrm{M}_{\text {pieds }}=3,54(\sigma=1,90), \mathrm{t}(34)=1,69 \mathrm{p}=0,100\right)$ alors que les autres attributs ont des moyennes autour de 5 (nombre de tiroirs, hauteur, couleur et essence de bois). Le type de vis et le type de pieds sont donc retenus.

Il s'agit ensuite de mettre en évidence deux modalités pour chaque attribut afin de compléter l'expérimentation utilisant les analyses conjointes. L'objectif est de vérifier que les modalités sont évaluées de manière équivalente. Deux échelles d'Osgood à 7 échelons sont utilisées pour différencier la préférence pour les pieds carrés ou ronds d'un bureau et la préférence pour les vis à tête creuse ou à tête ronde. Le test $\mathrm{t}$ indique que la moyenne n'est pas significativement différente de 4 (le milieu de l'échelle) pour les vis $\left(\mathrm{M}_{\text {(creuse } v \text { s. ronde) }}=4,17 \quad(\sigma=1,79), t(34)=0,57\right.$ $\mathrm{p}=0,575)$ et que la moyenne n'est pas différente de 4,2 pour les pieds de bureau ( $\mathbf{M}_{\text {(rond vs. car- }}$ ré) $=4,69(\sigma=1,97), t(34)=1,46 \mathrm{p}=0,153)$. Les deux attributs, type de vis (rondes ou creuses) et type de pieds (carrés ou ronds), sont ajoutés aux attributs du bureau à évaluer en expérimentation 2.

\section{A4.2. Vérification de manipulation des attributs neutres}

Importance du type de vis et du type de pieds : les tests t indiquent que la moyenne est significativement inférieure à 4 (milieu de l'échelle) pour l'importance du type de vis $\left(\mathrm{M}_{\mathrm{vis}}=3,14\right.$ $(\sigma=1,73) \mathrm{t}(168)=-6,45 \mathrm{p}<0,0001)$ et n'est pas différente de 4,2 pour l'importance du type de pieds $\left(\mathrm{M}_{\text {pieds }}=4,36(\sigma=1,77) \mathrm{t}(168)=1,18 \mathrm{p}=0,239\right)$.

Préférences pour un type de vis ou pour un type de pieds: les tests $\mathrm{t}$ indiquent que les moyennes des échelles de préférence (utilisant un différentiel sémantique à 7 échelons entre les 2 modalités) ne sont pas différentes de 4 pour les vis $\left(M_{\text {(creuse } v \text {. ronde) }}=3,96(\sigma=1,31) t(168)=-0,35\right.$ $\mathrm{p}=0,725)$ et de 4,2 pour les pieds $\left(\mathrm{M}_{\text {(ronds vs. carrés) }}=4,34(\sigma=1,80) \mathrm{t}(168)=0,99 \mathrm{p}=0,324\right)$. 


\section{A4.3. Autres explications possibles}

La désirabilité sociale n'a pas d'effet direct sur le poids des attributs environnementaux (émission de $\mathrm{C} 02$ : $\mathrm{t}(112)=-0,10 \mathrm{p}=0,917$; matière recyclée : $\mathrm{t}(109)=-0,55 \mathrm{p}=0,587)$ ni d'effet d'interaction avec la condition «nombre d'attributs environnementaux» (émission de CO2 : $\mathrm{t}(112)=-0,85 \mathrm{p}=0,397 \quad \mathrm{R}^{2}=0,04$; matière recyclée: $\left.\mathrm{t}(109)=-0,08 \quad \mathrm{p}=0,933 \quad \mathrm{R}^{2}=0,03\right)$. L'insensibilité au nombre d'attributs environnementaux n'est donc pas expliquée par la désirabilité sociale dans cette étude.

L'attente envers la qualité a un effet positif direct significatif sur le poids des deux attributs environnementaux (émission de $\mathrm{CO} 2: \mathrm{t}(112)=3,18 \mathrm{p}=0,0019$; matière recyclée : $\mathrm{t}(109)=1,90$ $\mathrm{p}=0,0597)$. Ce résultat indique que les individus sensibles à la qualité, donneront un plus grand poids aux meilleurs niveaux des attributs environnementaux. Le test d'interaction de l'attente envers la qualité avec le nombre d'attributs sur le poids des attributs environnementaux n'est statistiquement significatif pour aucun attribut (émission de CO2 : $t(112)=-1,41 \mathrm{p}=0,160$ $\mathrm{R}^{2}=0,13$; matière recyclée : $\left.\mathrm{t}(109)=-1,01 \mathrm{p}=0,317 \mathrm{R}^{2}=0,07\right)$. L'attente envers la qualité ne semble donc pas expliquer l'effet d'inclusion dans cette étude.

Corrélation «émission de CO2 - matière recyclée » : aucun effet direct ni effet d'interaction statistiquement significatif n'est mis en évidence pour la corrélation déclarée entre un haut pourcentage de réduction de $\mathrm{CO} 2$ et un important contenu de matière recyclée sur le poids attribué aux attributs environnementaux (Tableau A4a).

\section{(Insérer Tableau A4a)}

Corrélation «matière recyclée - émission de CO2» : aucun effet direct ni effet d'interaction n'est mis en évidence pour la corrélation déclarée entre un important contenu de matière recyclée et un pourcentage élevé de réduction de $\mathrm{CO} 2$ sur le poids attribué aux attributs environnementaux (Tableau A4b). Les corrélations déclarées par les consommateurs entre les deux attributs n'expliquent pas non plus l'effet d'inclusion.

\section{(Insérer Tableau A4b)}

Dans la même idée, la corrélation mesurée entre les attributs environnementaux en condition A n'est pas statistiquement significative $\left(r_{p}=0,101 p=0,459\right)$. L'effet d'inclusion n'est donc pas sensible à la corrélation mesurée entre les deux attributs environnementaux, contrairement aux observations de l'expérimentation 1. 
Tableau 1. - Synthèse de la littérature sur l'effet d'inclusion

\begin{tabular}{|c|c|c|c|c|c|}
\hline $\mathbf{n}^{\circ}$ & Définitions & Auteurs & Domaine & Mesure d'évaluation & Stimulus \\
\hline 1 & $\begin{array}{l}\text { «Le même bien se voit attribuer une valeur inférieure si le consen- } \\
\text { tement à payer pour lui est déduit du consentement à payer pour un } \\
\text { bien plus large plutôt que si le bien donné est évalué seul »p.58 }\end{array}$ & $\begin{array}{l}\text { Kahneman et } \\
\text { Knetsch } \\
(1992 \mathrm{a})\end{array}$ & Économie & - Consentement à payer & $\begin{array}{l}\text { - Programmes environ- } \\
\text { nementaux }\end{array}$ \\
\hline 2 & $\begin{array}{l}\text { «Cet effet }[. . .] \text { se produit lorsque le consentement à payer pour un } \\
\text { bien s'avère être non significativement différent du consentement à } \\
\text { payer pour un bien plus large. Ce dernier bien peut être plus large à } \\
\text { l'égard de la couverture géographique, du temps, ou tout autre attri- } \\
\text { but » p. } 248\end{array}$ & $\begin{array}{l}\text { Harrison } \\
(1992)\end{array}$ & Économie & \multicolumn{2}{|c|}{ Critique du travail de Kahneman et Knetsch (1992a } \\
\hline 3 & $\begin{array}{c}\text { «L'observation que les gens sont apparemment prêts à payer le } \\
\text { même montant d'argent pour un bien que pour une partie de ce bien » } \\
\text { p. } 211\end{array}$ & $\begin{array}{l}\text { Fischhoff et } \\
\text { alii (1993) }\end{array}$ & Économie & $\begin{array}{l}\text { - Consentement à payer } \\
\text { - Choix }\end{array}$ & $\begin{array}{l}\text { - Programmes environ- } \\
\text { nementaux }\end{array}$ \\
\hline 4 & $\begin{array}{l}\text { «Lorsque les éléments constitutifs d'un ensemble sont évalués sépa- } \\
\text { rément, la somme de ces évaluations a tendance à dépasser la valeur } \\
\text { placée dans l'ensemble »p.322 }\end{array}$ & $\begin{array}{l}\text { Bateman et alii } \\
\text { (1997) }\end{array}$ & Économie & $\begin{array}{l}\text { - Consentement à payer } \\
\text { - Consentement à accepter }\end{array}$ & $\begin{array}{l}\text { - Alimentaire (plat, des- } \\
\text { sert, menu) }\end{array}$ \\
\hline 5 & $\begin{array}{l}\text { «Renvoie au fait que parfois deux ou plusieurs produits environne- } \\
\text { mentaux sont évalués moins bien ensemble que séparément » p.340 }\end{array}$ & $\begin{array}{l}\text { Irwin et Spira } \\
\qquad(1997)\end{array}$ & Marketing & $\begin{array}{l}\text { - Consentement à payer } \\
\text { - Probabilité d'achat } \\
\text { - Environnementalisme } \\
\text { perçu }\end{array}$ & $\begin{array}{l}\text { - Voiture (échappement } \\
\text { de CO2, contenu recy- } \\
\text { clé) }\end{array}$ \\
\hline 6 & $\begin{array}{l}\text { «Ceci apparait lorsque le consentement à payer pour un bien parti- } \\
\text { culier n' est pas différent du consentement à payer pour un bien plus } \\
\text { large dont il fait partie (dans lequel il est inclus)»p. } 252\end{array}$ & $\begin{array}{l}\text { Shiell et Gold } \\
\text { (2002) }\end{array}$ & Économie & - Consentement à payer & - Santé (vaccin) \\
\hline 7 & $\begin{array}{l}\text { «Les répondants semblent donner la même valeur à un programme } \\
\text { indépendamment de s'il est large ou étroit »p.446 }\end{array}$ & $\begin{array}{c}\text { Olsen, } \\
\text { Donaldson et } \\
\text { Pereira (2004). }\end{array}$ & Économie & - Consentement à payer & $\begin{array}{l}\text { - Programme de santé } \\
\text { (nombre de patients } \\
\text { sauvés) }\end{array}$ \\
\hline 8 & $\begin{array}{c}\text { «Insensibilité à l'échelle ou à la grandeur - lorsque les répondants } \\
\text { donnent un consentement à payer très proche pour des niveaux diffé- } \\
\text { rents d'avantages » p.1428 }\end{array}$ & $\begin{array}{c}\text { Baker, Robin- } \\
\text { son et Smith } \\
(2008)\end{array}$ & Économie & Méta-analyse & \\
\hline 9 & $\begin{array}{l}\text { «C'est le phénomène qui apparait lorsque le consentement à payer } \\
\text { donné par les individus pour un bien plus large diffère du consente- } \\
\text { ment à payer agrégé obtenu des sous-parties du bien évaluées indé- } \\
\text { pendamment » p.1171 }\end{array}$ & $\begin{array}{l}\text { Jacobsen et alii } \\
\text { (2011) }\end{array}$ & Économie & $\begin{array}{l}\text { - Choix entre différents } \\
\text { montants de taxes à payer }\end{array}$ & $\begin{array}{l}\text { - Programmes environ- } \\
\text { nementaux }\end{array}$ \\
\hline
\end{tabular}


Tableau 2. - Poids des attributs environnementaux selon la condition dans les expérimentations 1 et 2 (les écart-types sont notés entre parenthèse)

\begin{tabular}{|c|c|c|c|c|c|}
\hline & Condition & $\mathbf{n}$ & $\begin{array}{c}\text { Attribut } 1 \\
M_{1}=\frac{1}{n} \sum_{i=1}^{\mathrm{n}} b 1_{i}\end{array}$ & $\begin{array}{c}\text { Attribut } 2 \\
M_{2}=\frac{1}{n} \sum_{\mathrm{i}=1}^{\mathrm{n}} b 2_{i}\end{array}$ & $\begin{array}{c}\text { Attribut } 3 \\
M_{3}=\frac{1}{n} \sum_{\mathrm{i}=1}^{\mathrm{n}} b 3_{i}\end{array}$ \\
\hline & & & Écolabel & Marque envi ${ }^{\text {tale }}$ & Prix \\
\hline Ex. 1 & $\begin{array}{l}\text { A - } 2 \text { attributs } \\
\text { B - Écolabel } \\
\text { C - Marque }\end{array}$ & $\begin{array}{l}56 \\
58 \\
59\end{array}$ & $\begin{array}{l}\mathrm{M}_{\mathrm{EA}}=0,38(0,64) \\
\mathrm{M}_{\mathrm{EB}}=0,66(0,68)\end{array}$ & $\mathrm{M}_{\mathrm{MC}}=0,36(0,71)$ & $\begin{array}{l}\mathrm{M}_{\mathrm{PA}}=0,95(1,00) \\
\mathrm{M}_{\mathrm{PB}}=1,68(0,85) \\
\mathrm{M}_{\mathrm{PC}}=1,54(0,77)\end{array}$ \\
\hline & & & $\begin{array}{c}\mathrm{CO2+} \\
\text { Réduction élevée } \\
\text { d'émission de } \mathrm{C02}\end{array}$ & $\begin{array}{c}\text { recy+ } \\
\text { \% élevé de matière } \\
\text { Recyclée }\end{array}$ & Prix \\
\hline Ex. 2 & $\begin{array}{l}\text { A - } 2 \text { attributs } \\
\text { B - CO2 } \\
\text { C - mat. Recy. }\end{array}$ & $\begin{array}{l}58 \\
55\end{array}$ & $\begin{array}{l}\mathrm{M}_{\mathrm{CA}}=0,33(0,41) \\
\mathrm{M}_{\mathrm{CB}}=0,53(0,64)\end{array}$ & $\mathrm{M}_{\mathrm{RC}}=0,52(0,57)$ & $\begin{array}{l}\mathrm{M}_{\mathrm{PA}}=0,95(0,93) \\
\mathrm{M}_{\mathrm{PB}}=1,01(0,94) \\
\mathrm{M}_{\mathrm{PC}}=1,15(1,15)\end{array}$ \\
\hline
\end{tabular}


TABLEAU A1a - Options présentées dans les trois conditions (expérimentation 1)

\begin{tabular}{|c|c|c|c|c|}
\hline \multirow{4}{*}{ Condition A } & option 1 & option 2 & option 3 & option 4 \\
\hline & $\begin{array}{l}\text { Proverde } \\
\text { PEFC } \\
\$ 350\end{array}$ & $\begin{array}{l}\text { Proverde } \\
\text { PEFC } \\
\$ 550\end{array}$ & $\begin{array}{l}\text { Proverde } \\
\text { pas PEFC } \\
\$ 350\end{array}$ & $\begin{array}{l}\text { Proverde } \\
\text { pas PEFC } \\
\$ 550\end{array}$ \\
\hline & option 5 & option 6 & option 7 & option 8 \\
\hline & $\begin{array}{l}\text { Beavara } \\
\text { PEFC } \\
\$ 350\end{array}$ & $\begin{array}{l}\text { Beavara } \\
\text { PEFC } \\
\$ 550\end{array}$ & $\begin{array}{l}\text { Beavara } \\
\text { pas PEFC } \\
\$ 350\end{array}$ & $\begin{array}{l}\text { Beavara } \\
\text { pas PEFC } \\
\$ 550\end{array}$ \\
\hline \multirow[b]{2}{*}{ Condition B } & option 1 & option 2 & option 3 & option 4 \\
\hline & $\begin{array}{l}\text { PEFC } \\
\$ 350\end{array}$ & $\begin{array}{l}\text { PEFC } \\
\$ 550\end{array}$ & $\begin{array}{l}\text { pas PEFC } \\
\$ 350\end{array}$ & $\begin{array}{l}\text { pas PEFC } \\
\$ 550\end{array}$ \\
\hline \multirow[b]{2}{*}{ Condition $\mathbf{C}$} & option 1 & option 2 & option 3 & option 4 \\
\hline & $\begin{array}{l}\text { Proverde } \\
\$ 350\end{array}$ & $\begin{array}{l}\text { Proverde } \\
\$ 550\end{array}$ & $\begin{array}{l}\text { Beavara } \\
\$ 350\end{array}$ & $\begin{array}{l}\text { Beavara } \\
\$ 550\end{array}$ \\
\hline
\end{tabular}




\section{TABLEAU A1b - Options présentées dans les trois conditions (expérimentation 2)}

Le type de vis (tête ronde $v s$. tête creuse) et le type de pieds (ronds vs. carrés) complètent de manière aléatoire les options présentées.

\begin{tabular}{|c|c|c|c|c|}
\hline \multirow{4}{*}{$\begin{array}{c}\text { Condition } \\
\text { A }\end{array}$} & option 1 & option 2 & option 3 & option 4 \\
\hline & $\begin{array}{l}\text { - } 21 \% \text { de bois recy- } \\
\text { clé } \\
\text { - } 25 \% \text { d'émission de } \\
\text { CO2 en moins } \\
\text { - Pieds ronds } \\
\text { - Tête de vis creuses } \\
\text { - } \$ 350\end{array}$ & $\begin{array}{l}\text { - } 21 \% \text { de bois recy- } \\
\text { clé } \\
\text { - } 25 \% \text { d'émission de } \\
\text { CO2 en moins } \\
\text { - Pieds carrés } \\
\text { - Tête de vis rondes } \\
\text { - } \$ 550\end{array}$ & $\begin{array}{l}\text { - } 21 \% \text { de bois recy- } \\
\text { clé } \\
\text { - } 10 \% \text { d'émission de } \\
\mathrm{CO} 2 \text { en moins } \\
\text { - Pieds ronds } \\
\text { - Tête de vis creuses } \\
\text { - } \$ 350\end{array}$ & $\begin{array}{l}\text { - } 21 \% \text { de bois recy- } \\
\text { clé } \\
\text { - } 10 \% \text { d'émission de } \\
\text { CO2 en moins } \\
\text { - Pieds carrés } \\
\text { - Tête de vis rondes } \\
\text { - } \$ 550\end{array}$ \\
\hline & option 5 & option 6 & option 7 & option 8 \\
\hline & $\begin{array}{l}\text { - } 14 \% \text { de bois recy- } \\
\text { clé } \\
\text { - } 25 \% \text { d'émission de } \\
\text { CO2 en moins } \\
\text { - Pieds ronds } \\
\text { - Tête de vis rondes } \\
\text { - } \$ 350\end{array}$ & $\begin{array}{l}\text { - } 14 \% \text { de bois recy- } \\
\text { clé } \\
\text { - } 25 \% \text { d'émission de } \\
\text { CO2 en moins } \\
\text { - Pieds carrés } \\
\text { - Tête de vis creuses } \\
\text { - } \$ 550\end{array}$ & $\begin{array}{l}\text { - } 14 \% \text { de bois recy- } \\
\text { clé } \\
\text { - } 10 \% \text { d'émission de } \\
\text { CO2 en moins } \\
\text { - Pieds ronds } \\
\text { - Tête de vis creuses } \\
\text { - } \$ 350\end{array}$ & $\begin{array}{l}\text { - } 14 \% \text { de bois recy- } \\
\text { clé } \\
\text { - } 10 \% \text { d'émission de } \\
\text { CO2 en moins } \\
\text { - Pieds carrés } \\
\text { - Tête de vis rondes } \\
\text { - } \$ 550\end{array}$ \\
\hline \multirow{4}{*}{$\begin{array}{c}\text { Condition } \\
\text { B }\end{array}$} & option 1 & option 2 & option 3 & option 4 \\
\hline & $\begin{array}{l}\text { - } 25 \% \text { d'émission de } \\
\text { CO2 en moins } \\
\text { - Pieds ronds } \\
\text { - Tête de vis creuses } \\
\text { - } \$ 350\end{array}$ & $\begin{array}{l}\text { - } 25 \% \text { d'émission de } \\
\text { CO2 en moins } \\
\text { - Pieds carrés } \\
\text { - Tête de vis rondes } \\
\text { - } \$ 550\end{array}$ & $\begin{array}{l}\text { - } 10 \% \text { d'émission de } \\
\text { CO2 en moins } \\
\text { - Pieds ronds } \\
\text { - Tête de vis creuses } \\
\text { - } \$ 350\end{array}$ & $\begin{array}{l}\text { - } 10 \% \text { d'émission de } \\
\text { CO2 en moins } \\
\text { - Pieds carrés } \\
\text { - Tête de vis rondes } \\
\text { - } \$ 550\end{array}$ \\
\hline & option 5 & option 6 & option 7 & option 8 \\
\hline & $\begin{array}{l}\text { - } 25 \% \text { d'émission de } \\
\text { CO2 en moins } \\
\text { - Pieds carrés } \\
\text { - Tête de vis rondes } \\
\text { - } \$ 350\end{array}$ & $\begin{array}{l}\text { - } 25 \% \text { d'émission de } \\
\text { CO2 en moins } \\
\text { - Pieds ronds } \\
\text { - Tête de vis creuses } \\
\text { - } \$ 550\end{array}$ & $\begin{array}{l}\text { - } 10 \% \text { d'émission de } \\
\text { CO2 en moins } \\
\text { - Pieds carrés } \\
\text { - Tête de vis creuses } \\
\text { - } \$ 350\end{array}$ & $\begin{array}{l}\text { - } 10 \% \text { d'émission de } \\
\text { CO2 en moins } \\
\text { - Pieds ronds } \\
\text { - Tête de vis rondes } \\
\text { - } \$ 550\end{array}$ \\
\hline \multirow{4}{*}{$\begin{array}{c}\text { Condition } \\
\text { C }\end{array}$} & option 1 & option 2 & option 3 & option 4 \\
\hline & $\begin{array}{l}\text { - } 21 \% \text { de bois recy- } \\
\text { clé } \\
\text { - Pieds ronds } \\
\text { - Tête de vis creuses } \\
\text { - } \$ 350\end{array}$ & $\begin{array}{l}\text { - } 21 \% \text { de bois recy- } \\
\text { clé } \\
\text { - Pieds carrés } \\
\text { - Tête de vis rondes } \\
\text { - } \$ 550\end{array}$ & $\begin{array}{l}\text { - } 14 \% \text { de bois recy- } \\
\text { clé } \\
\text { - Pieds ronds } \\
\text { - Tête de vis creuses } \\
\text { - } \$ 350\end{array}$ & $\begin{array}{l}\text { - } 14 \% \text { de bois recy- } \\
\text { clé } \\
\text { - Pieds carrés } \\
\text { - Tête de vis rondes } \\
\text { - } \$ 550\end{array}$ \\
\hline & option 5 & option 6 & option 7 & option 8 \\
\hline & $\begin{array}{l}\text { - } 21 \% \text { de bois recy- } \\
\text { clé } \\
\text { - Pieds carrés } \\
\text { - Tête de vis rondes } \\
\text { - } \$ 350\end{array}$ & $\begin{array}{l}\text { - } 21 \% \text { de bois recy- } \\
\text { clé } \\
\text { - Pieds ronds } \\
\text { - Tête de vis creuses } \\
\text { - } \$ 550\end{array}$ & $\begin{array}{l}\text { - } 14 \% \text { de bois recy- } \\
\text { clé } \\
\text { - Pieds carrés } \\
\text { - Tête de vis creuses } \\
\text { - } \$ 350\end{array}$ & $\begin{array}{l}\text { - } 14 \% \text { de bois recy- } \\
\text { clé } \\
\text { - Pieds ronds } \\
\text { - Tête de vis rondes } \\
\text { - } \$ 550\end{array}$ \\
\hline
\end{tabular}


TABLEAU A2 - Fiabilité, validité convergente et validité discriminante des échelles de l'expérimentation 2

\begin{tabular}{|c|c|c|c|c|c|}
\hline & & \multicolumn{3}{|c|}{ Carré des corrélations } & Modèle testé \\
\hline Échelles & $\rho_{\mathrm{vc}}$ & $\begin{array}{c}\text { Att. Qua- } \\
\text { lité }\end{array}$ & $\begin{array}{c}\text { Impli. } \\
\text { Écolabel }\end{array}$ & PPE & \multirow{4}{*}{ 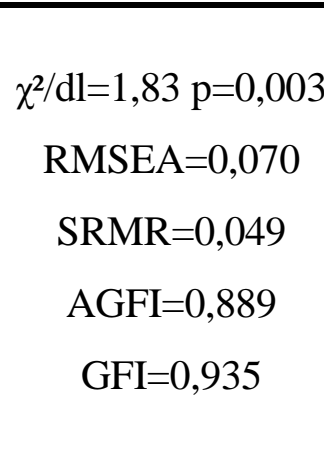 } \\
\hline Att. Qualité & $0,42^{(a)}$ & $0,65^{(b)}$ & & & \\
\hline Impli. Écolabel & $\mathbf{0 , 7 5}$ & $0,07^{(\mathrm{c})}$ & 0,92 & & \\
\hline PPE & 0,79 & 0,11 & 0,51 & 0,91 & \\
\hline
\end{tabular}

(a) La variance extraite moyenne ( $\left.\rho_{v c}\right)$ par construit est présentée dans la deuxième colonne, en gras.

(b) Les coefficients alpha sont présentés dans la diagonale, en italique.

(c) Les carrés des corrélations entre construits $\left(\phi^{2}\right)$ sont présentés dans le triangle inférieur. 
TABLEAU A4a - Effets direct et d'interaction de la corrélation «émission de CO2matière recyclée » sur le poids des attributs environnementaux

\begin{tabular}{c|c|c|c}
\hline \multicolumn{1}{c|}{ Corrélation déclarée } & Poids des attributs & Effet direct & Effet d'interaction \\
\hline \multirow{2}{*}{ Émission CO2 - Matière recyclée } & $\mathrm{CO} 2$ & $\mathrm{t}(112)=-0,67 \mathrm{p}=0,502$ & $\mathrm{t}(112)=-0,44 \mathrm{p}=0,657$ \\
\cline { 2 - 4 } & Matière recyclée & $\mathrm{t}(109)=-0,10 \mathrm{p}=0,922$ & $\mathrm{t}(109)=-0,60 \mathrm{p}=0,551$ \\
\hline
\end{tabular}


TABLEAU A4b - Effets direct et d'interaction de la corrélation « matière recyclée - émission de $\mathrm{CO} 2$ » sur le poids des attributs environnementaux

\begin{tabular}{c|c|c|c}
\hline Corrélation déclarée & Poids des attributs & Effet direct & Effet d'interaction \\
\hline \multirow{2}{*}{ Matière recyclée - Émission CO2 } & $\mathrm{CO} 2$ & $\mathrm{t}(112)=0,24 \mathrm{p}=0,808$ & $\mathrm{t}(112)=-1,16 \mathrm{p}=0,248$ \\
\cline { 2 - 4 } & Matière recyclée & $\mathrm{t}(109)=0,64 \mathrm{p}=0,524$ & $\mathrm{t}(109)=0,80 \mathrm{p}=0,425$ \\
\hline
\end{tabular}


Figure 1. - Le modèle de recherche

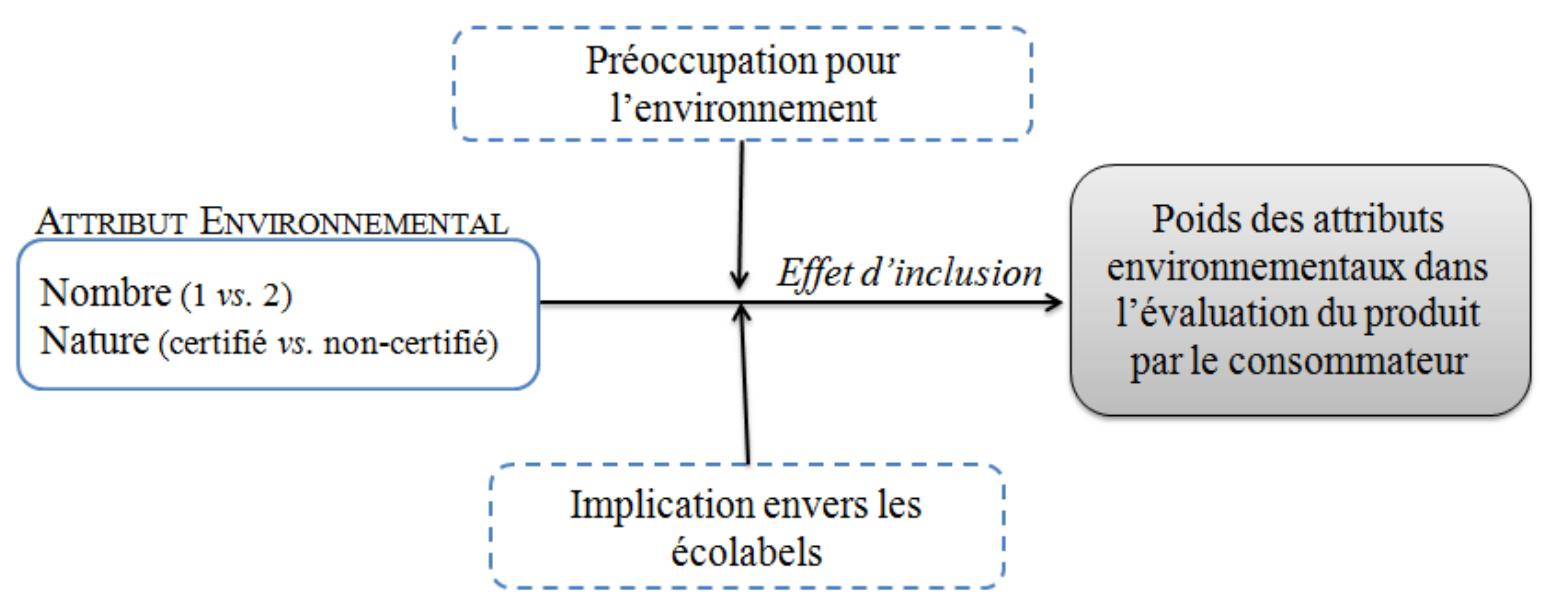

Variables explicatives contrôlées

- Désirabilité sociale

- Attente envers la qualité du produit

- Corrélations perçues et mesurées entre les attributs environnementaux 
Figure 2. - Moyennes des poids des attributs environnementaux selon le nombre d'attributs - Expérimentation 1

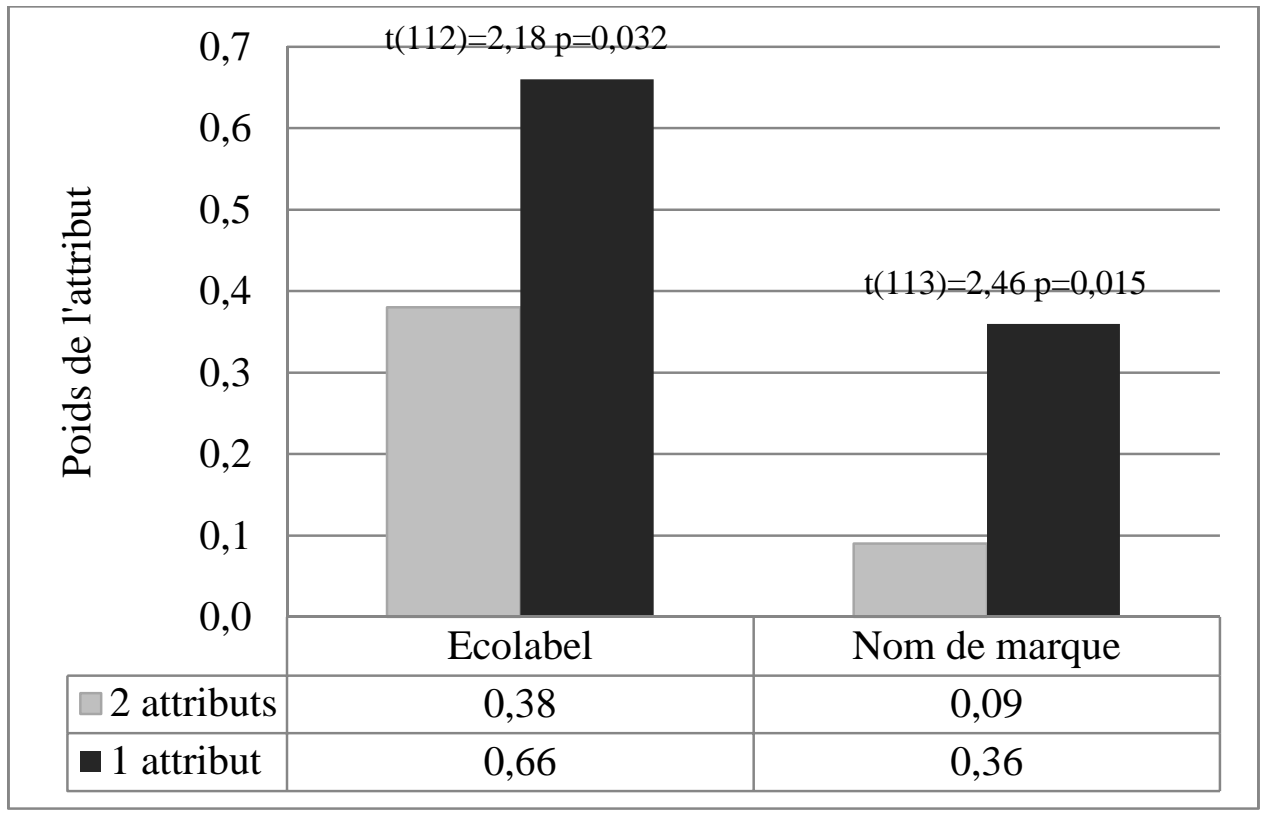


Figure 3. - Interaction entre le nombre d'attributs et la PPE sur le poids du nom de marque - Expérimentation 1

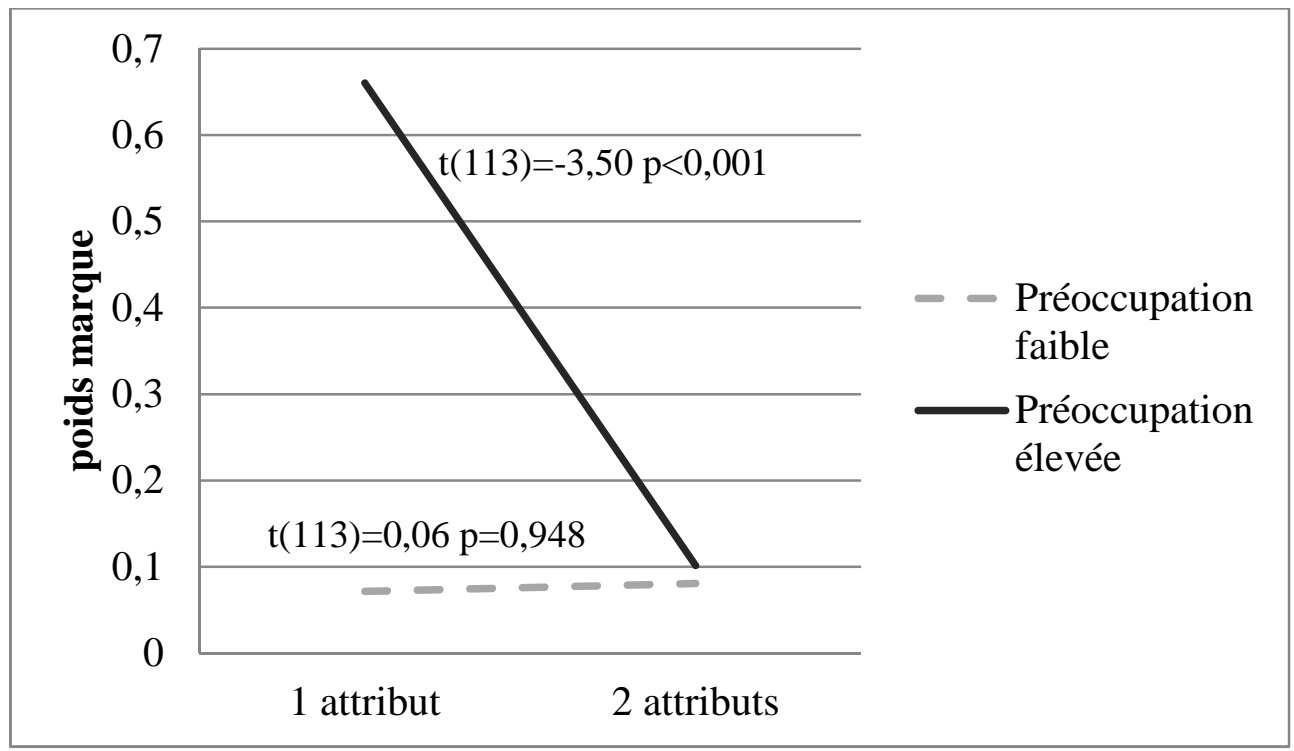


Figure 4. - Moyennes des poids des attributs environnementaux selon le nombre d'attributs - Expérimentation 2

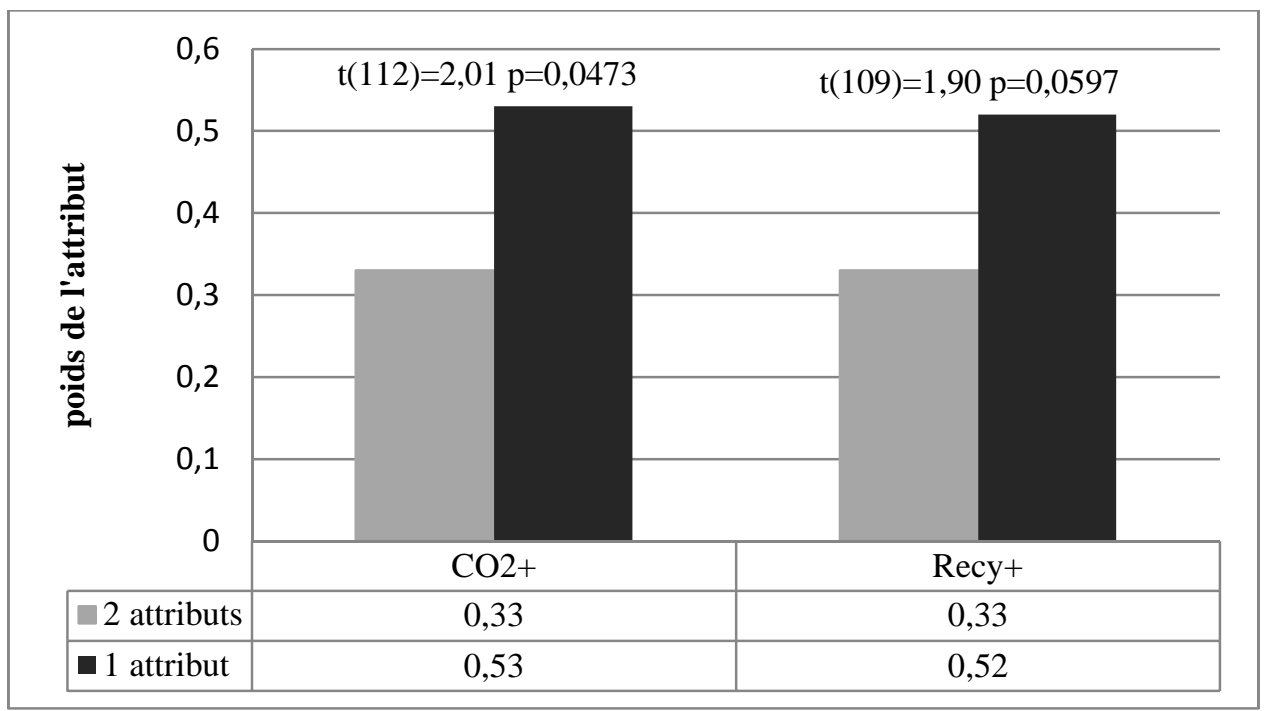


Figure 5. - Interaction entre le nombre d'attributs et la PPE sur le poids du label C02+ Expérimentation 2

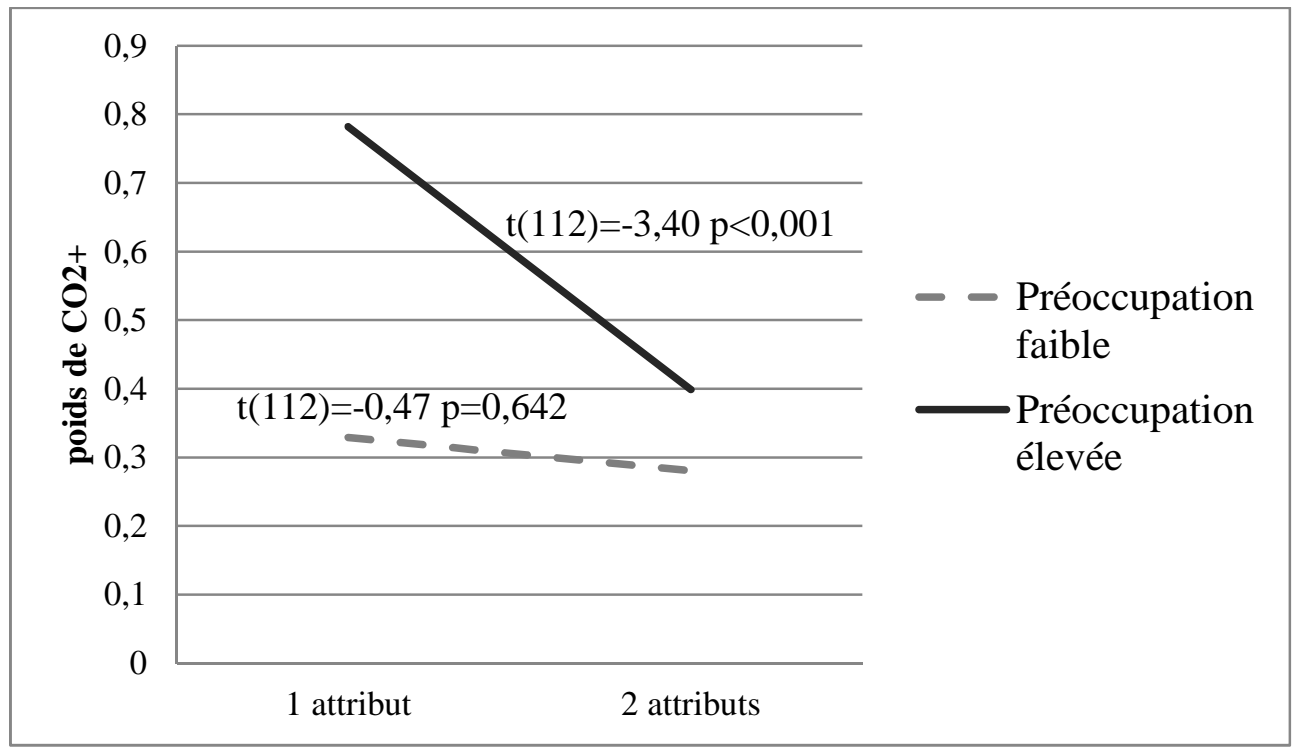


Figure 6. - Interaction entre le nombre d'attributs et l'implication envers les écolabels sur le poids du label $\mathrm{CO} 2+$ - Expérimentation 2

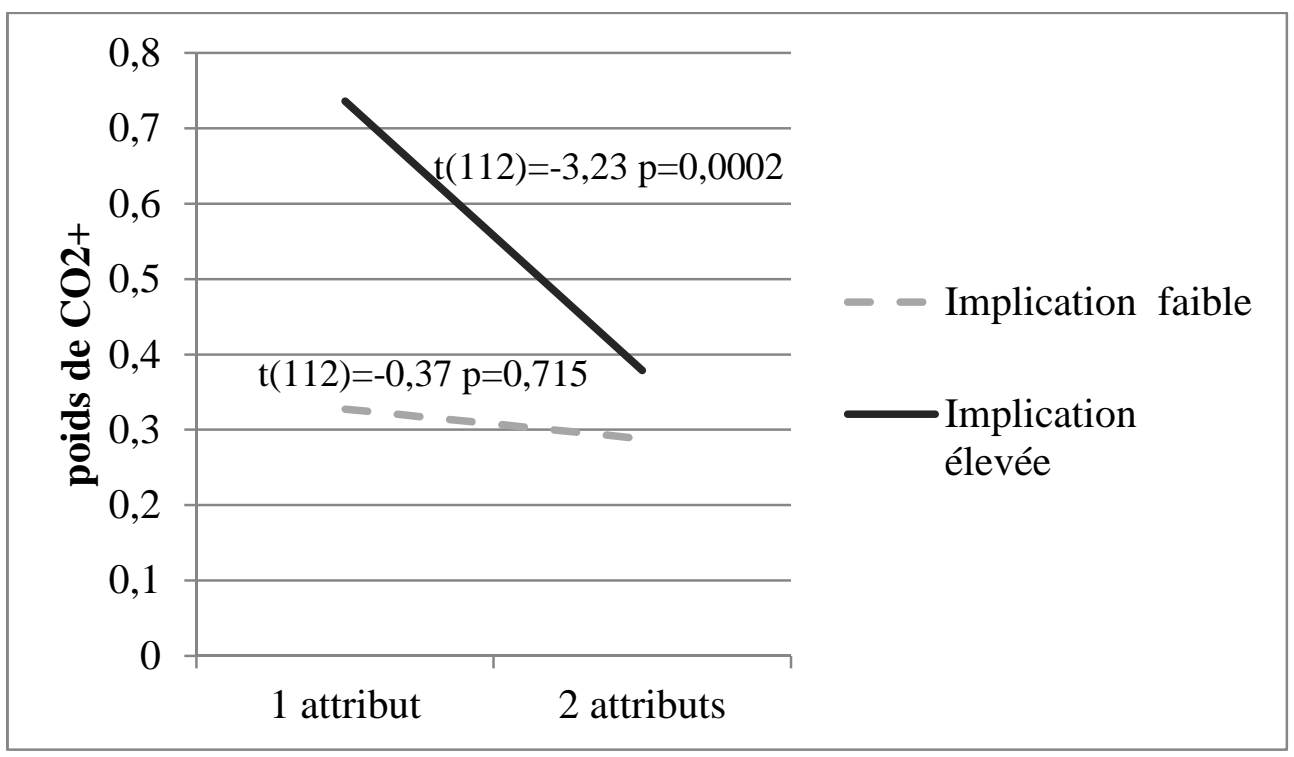




\section{RÉFÉRENCES BIBLIOGRAPHIQUES}

Allenby G., Fennell G., Huber J., Eagle T., Gilbride T., Horsky D., Kim J., Lenk P., Johnson R., Ofek E., Orme B., Otter T et Walker J. (2005), Adjusting choice models to better predict market behavior, Marketing Letters, 16, 3-4, 197-208.

Andreoni J. (1989), Giving with impure altruism: applications to charity and Ricardian equivalence, Journal of Political Economy, 97, 6, 1447-1458.

Andreoni J. (1990), Impure altruism et donations to public goods: a theory of warm-glow giving, Economic Journal, 100, 401, 464-477.

Baker R., Robinson A. et Smith R. (2008), How do respondents explain WTP responses? A review of the qualitative evidence, Journal of Socio-Economics, 37, 4, 1427-1442.

Baron J. et Ritov I. (2009), Protected values and omission bias as deontological judgments, in D. Bartels, C. Bauman L. Skitka et D. Medin (coord.), Moral Judgment and Decision Making, San Diego, Academic Press, 133-167.

Baron J. et Spranca M. (1997), Protected values, Organizational Behavior and Human Decision Processes, 70, 1, 1-16.

Bateman I., Munro A., Rhodes B., Starmer C. et Sugden R. (1997), Does part-whole bias exist? an experimental investigation, Economic Journal, 107, 441, 322-332.

Bettman J., Luce M.-F. et Payne J. (1998), Constructive consumer choice processes, Journal of Consumer Research, 25, 3, 187-217.

Bougherara D. et Piguet V. (2008), Marchés avec coûts d'information sur la qualité des biens: une application aux produits écolabellisés, Économie \& Prévision, 182, 1, 77-96.

Brécard D., Hlaimi B., Lucas S., Perraudeau Y. et Salladarré F. (2009), Determinants of demand for green products: an application to eco-label demand for fish in Europe, Ecological Economics, 69, 1, 115-125.

Carson R. (1997), Contingent valuation surveys and tests of insensitivity to scope, in R. Kopp, W. Pommerhene, et N. Schwartz (coord.), Determining the value of non-marketed goods: economic, psychological, and policy relevant aspects of contingent valuation methods, Boston, Kluwer, 127-164.

Chernev A. et Gal D. (2010), Categorization effects in value judgments: averaging bias in evaluating combinations of vices and virtues, Journal of Marketing Research, 47, 4, 738747.

Chumpitaz R. et Vanhamme J. (2003), Les processus modérateurs et médiateurs: distinction conceptuelle, aspects analytiques et illustrations, Recherche et Applications en Marketing, 
$18,2,67-100$.

Cohen J. et Basu K. (1987), Alternative models of categorization: Toward a contingent processing framework, Journal of Consumer Research, 13, 4, 455-472.

Comas J. et Seifert R. (2012), Reviewing the adoption of ecolabels by firms, A Survey Report, EPFL - IMD International, Switzerland, EPFL - IMD, 23.

Darby M. et Karni E. (1973), Free competition and the optimal amount of fraud, Journal of Law and Economics, 16, 1, 67-88.

Desvousges W., Johnson F., Dunford R., Boyle K., Hudson S. et Wilson K. (1992), Measuring nonuse damages using contingent valuation: an experimental evaluation of accuracy, NC, RTI International.

Dietz T., Kalof L. et Stern P. (2002), Gender, values, and environmentalism, Social Science Quarterly, 83, 1, 353-364.

Ehrich K. et Irwin J. (2005), Willful ignorance in the request for product attribute information, Journal of Marketing Research, 42, 3, 266-277.

Einhorn H. et Hogarth R. (1981), Behavioral decision theory: processes of judgment and choice, Journal of Accounting Research, 32, 1, 53-88.

Fishbein M. et Ajzen I. (1975), Belief, attitude, intention, and behavior: an introduction to theory and research, Reading (MA), Addison-Wesley.

Fischhoff B., Quadrel M., Kamlet M., Loewenstein G., Dawes R., Fischbeck P., Klepper S., Leland J. et Stroh P. (1993), Embedding effects: stimulus representation and response mode, Journal of Risk and Uncertainty, 6, 3, 211-234.

Friedrich J., Barnes P., Chapin K., Dawson I., Garst V. et Kerr D. (1999), Psychophysical numbing: when lives are valued less as the lives at risk increase, Journal of Consumer Psychology, 8, 3, 277-299.

Giannelloni J.-L. (1998), Les comportements liés à la protection de l'environnement et leurs déterminants: un état des recherches en marketing, Recherche et Applications en Marketing, 13, 2, 49-72.

Grunert S. et Juhl H. (1995), Values, environmental attitudes, and buying of organic foods. Journal of Economic Psychology, 16, 1, 39-62.

Harrison G. (1992), Valuing public goods with the contingent valuation method: A critique of Kahneman and Knetsch, Journal of Environmental Economics and Management, 23, 3, $248-257$. 
Henion K. (1972), The effect of ecologically relevant information on detergent sales, Journal of Marketing Research, 9, 1, 10-14.

Hong J. et Sternthal B. (2010), The effects of consumer prior knowledge and processing strategies on judgments, Journal of Marketing Research, 47, 2, 301-311.

Huber J., Holbrook M. et Kahn B. (1986), Effects of competitive context and of additional information on price sensitivity, Journal of Marketing Research, 23, 3, 250-260.

Huber J. et McCann J. (1982), The impact of inferential beliefs on product evaluations, Journal of Marketing Research, 19, 3, 324-333.

Irwin J. (2009), Continuous variables : analyzing and interpreting interaction models, Présenté au SCP doctorial consortium, San Diego.

Irwin J. et Baron J. (2001), Response mode effects and moral values, Organizational Behavior and Human Decision Processes, 84, 2, 177-197.

Irwin J. et McClelland G. (2001), Misleading heuristics for moderated multiple regression models, Journal of Marketing Research, 35, 1, 100-109.

Irwin J. et McClelland G. (2003), Negative consequences of dichotomizing continuous predictor variables, Journal of Marketing Research, 40, 3, 366-371.

Irwin J. et Naylor R. (2009), Ethical decisions and response mode compatibility: weighting of ethical ettributes in consideration sets formed by excluding versus including product alternatives, Journal of Marketing Research, 46, 2, 234-246.

Irwin J. et Spira J. (1997), Anomalies in the values for consumer goods with environmental attributes, Journal of Consumer Psychology, 6, 4, 339-363.

Jacobsen J.-B., Lundhede T.-H., Martinsen L., Hasler B. et Thorsen B.-J. (2011), Embedding effects in choice experiment valuations of environmental preservation projects, Ecological Economics, 70, 6, 1170-1177.

Jolibert A. et Jourdan P. (2006), Marketing research: méthodes de recherche et d'études en marketing, Paris, Dunod.

Kahneman D. et Knetsch J. (1992a), Valuing public goods: the purchase of moral satisfaction, Journal of Environmental Economics and Management, 22, 1, 57-70.

Kahneman D. et Knetsch J. (1992b), Contingent valuation and the value of public goods: reply, Journal of Environmental Economics and Management, 22, 1, 90-94.

Kitira, V. (2009), Eco-labels: best practices and what's to come, présenté à l'EPA sustainable products network conference, webconférence.

Larceneux F., Benoit-Moreau F. et Renaudin V. (2011), Why might organic labels fail to influence consumer choices? Marginal labelling and brand equity effects, Journal of Consumer 
Policy, 35, 1, 85-104.

Lefkoff-Hagius R. et Mason C. (1993), Characteristic, beneficial, and image attributes in consumer judgments of similarity and preference, Journal of Consumer Research, 20, 1, $100-110$.

Le Gall-Ely M. et Robert-Demontrond P. (2005), Méthodes d'évaluation contingente et d'analyse conjointe, Paris, Apogée.

Loureiro M., McCluskey J. et Mittelhammer R. (2002), Will consumers pay a premium for ecolabeled apples ?, Journal of Consumer Affairs, 36, 2, 203-219.

Luchs M., Naylor R., Irwin J. et Rajagopal R. (2010), The sustainability liability: potential negative effects of ethicality on product preference, Journal of Marketing, 74, 5, 18-31.

Mazar N. et Zhong C-B. (2010), Do green products make us better people?, Psychological Science, 21, 4, 494-498.

Miller K., Hofstetter R., Krohmer H. et Zhang Z. (2011), How should consumers' willingness to pay be measured? an empirical comparison of state-of-the-art approaches, Journal of Marketing Research, 48, 1, $172-184$.

Nunnally J. (1978), Psychometric Theory, New York, McGraw-Hill.

Olsen J., Donaldson C. et Pereira J. (2004), The insensitivity of 'willingness-to-pay' to the size of the good: New evidence for health care, Journal of Economic Psychology, 25, 4, 445460.

Pham M.-T. (1996), Heuristiques et biais décisionnels en marketing, Recherche et Applications en Marketing, 11, 4, 53-69.

Rao A. et Bergen M. (1992), Price premium variations as a consequence of buyers' lack of information, Journal of Consumer Research, 19, 3, 412-423.

Rao A. et Monroe K. (1996), Causes and consequences of price premiums, Journal of Business, $69,4,511-535$.

Reynolds W. (1982), Development of reliable and valid short forms of the Marlowe-Crowne Social Desirability Scale, Journal of Clinical Psychology, 38, 1, 119-125.

Ritov I. et Baron J. (1999), Protected values and omission bias, Organizational Behavior and Human Decision Processes, 79, 2, 79-94.

Sedjo R. et Swallow S. (2002), Voluntary eco-labeling and the price premium, Land Economics, 78, 2, 272-284.

Shiell A. et Gold L. (2002), Contingent valuation in health care and the persistence of embedding effects without the warm glow, Journal of Economic Psychology, 23, 2, 251262. 
Smith V. (1992), Arbitrary values, good causes, and premature verdicts, Journal of Environmental Economics and Management, 22, 1, 71-89.

SNDD (2012), Mise en oeuvre de la stratégie nationale de développement durable 2010-2013, vers une economie verte et equitable, $2^{\mathrm{e}}$ rapport au Parlement, Paris.

Spiller S., Fitzsimons G., Lynch J. Jr. et McClelland G. (2013), Spotlights, floodlights, and the magic number zero: simple effects tests in moderated regression, Journal of Marketing Research, 50, 2, 277-288.

Steenkamp J.-B. (1989), Product quality: an investigation into the concept and how it is perceived by consumers, AssenMaastrich, Van Gorcum.

Steg L. et Vlek C. (2009), Encouraging pro-environmental behaviour: an integrative review and research agenda, Journal of Environmental Psychology, 29, 3, 309-317.

Strazzieri A. (1994), Mesurer l'implication durable vis-à-vis d'un produit indépendamment du risque perçu, Recherche et Applications en Marketing, 9, 1, 73-91.

Tellis G. et Gaeth G. (1990), Best value, price-seeking, and price aversion: the impact of information and learning on consumer choices, Journal of Marketing, 54, 2, 34-45.

TerraChoice (2010), The sins of greenwashing, home and family edition, rapport de UL, US.

Urminsky O. et Kivetz R. (2011), Scope insensitivity and the "mere token" effect, Journal of Marketing Research, 48, 2, 282-295.

Veisten K. (2007), Willingness to pay for eco-labelled wood furniture: choice-based conjoint analysis versus open-ended contingent valuation, Journal of Forest Economics, 13, 1, 29_ 48.

Vlosky R., Ozanne L. et Fontenot R. (1999), A conceptual model of US consumer willingnessto-pay for environmentally certified wood products, Journal of Consumer Marketing, 16, $2,122-136$.

White K., MacDonnell R. et Dahl D. (2011), It's the mind-set that matters : the role of construal level and message framing in influencing consumer efficacy and conservation behaviors, Journal of Marketing Research, 48, 3, 472-485.

Whitmarsh L. et O'Neill S. (2010), Green identity, green living? The role of pro-environmental self-identity in determining consistency across diverse pro-environmental behaviours, Journal of Environmental Psychology, 30, 3, 305-314.

Xiao C. et Dunlap R. (2007), Validating a comprehensive model of environmental concern cross-nationally: a U.S.-canadian comparison, Social Science Quarterly, 88, 2, 471-493.

Xu J., Jiang Z. et Dhar R. (2013), Mental representation and perceived similarity: how abstract mindset aids choice from large assortments, Journal of Marketing Research, 50, 1, 1-45. 